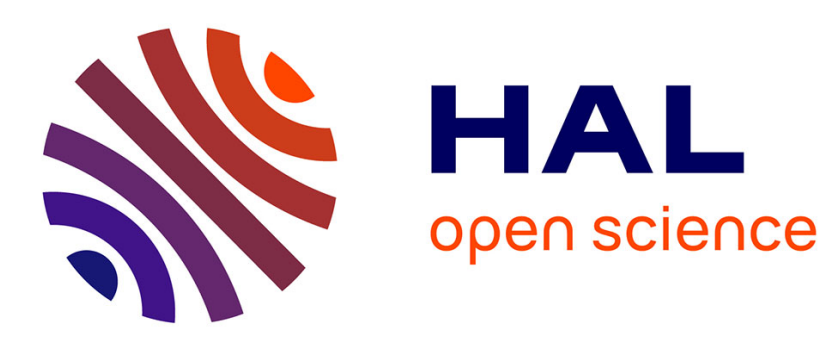

\title{
Librations of the Galilean satellites: the influence of global internal liquid layers
}

Rose-Marie Baland, Tim Van Hoolst

\section{To cite this version:}

Rose-Marie Baland, Tim Van Hoolst. Librations of the Galilean satellites: the influence of global internal liquid layers. Icarus, 2010, 209 (2), pp.651. 10.1016/j.icarus.2010.04.004 . hal-00676222

\section{HAL Id: hal-00676222 \\ https://hal.science/hal-00676222}

Submitted on 4 Mar 2012

HAL is a multi-disciplinary open access archive for the deposit and dissemination of scientific research documents, whether they are published or not. The documents may come from teaching and research institutions in France or abroad, or from public or private research centers.
L'archive ouverte pluridisciplinaire HAL, est destinée au dépôt et à la diffusion de documents scientifiques de niveau recherche, publiés ou non, émanant des établissements d'enseignement et de recherche français ou étrangers, des laboratoires publics ou privés. 


\section{Accepted Manuscript}

Librations of the Galilean satellites: the influence of global internal liquid layers

Rose-Marie Baland, Tim Van Hoolst

PII:

S0019-1035(10)00152-1

DOI:

10.1016/j.icarus.2010.04.004

Reference:

YICAR 9401

To appear in:

Icarus

Received Date: $\quad 25$ January 2010

Revised Date: $\quad 2$ April 2010

Accepted Date: $\quad 6$ April 2010

Please cite this article as: Baland, R-M., Hoolst, T.V., Librations of the Galilean satellites: the influence of global internal liquid layers, Icarus (2010), doi: 10.1016/j.icarus.2010.04.004

This is a PDF file of an unedited manuscript that has been accepted for publication. As a service to our customers we are providing this early version of the manuscript. The manuscript will undergo copyediting, typesetting, and review of the resulting proof before it is published in its final form. Please note that during the production process errors may be discovered which could affect the content, and all legal disclaimers that apply to the journal pertain. 


\title{
Librations of the Galilean satellites:
}

\section{the influence of global internal liquid layers}

\author{
Rose-Marie Baland ${ }^{a, b}$, Tim Van Hoolst ${ }^{a}$ \\ ${ }^{a}$ Royal Observatory of Belgium, Ringlaan 3, B-1180 Brussels, Belgium. \\ ${ }^{b}$ Université Catholique de Louvain, Earth and Life Institute, Georges Lemaître \\ Centre for Earth and Climate Research.
}

Number of manuscript pages: 65

Number of manuscript tables: 4

Number of manuscript figures: 9 
Proposed running head: Librations of the Galilean satellites

Editorial Correspondence to:

Rose-Marie Baland

Royal Observatory of Belgium

Ringlaan 3

B-1180 Brussels

Belgium

Email: Rose-Marie.Baland@oma.be

Phone: +322 7903928

Fax: +322 3749822 


\section{Abstract}

The four Galilean satellites are thought to harbour one or even two global internal liquid layers beneath their surface layer. The iron core of Io and Ganymede is most likely (partially) liquid and also the core of Europa may be liquid. Furthermore, there are strong indications for the existence of a subsurface ocean in Europa, Ganymede, and Callisto. Here, we investigate whether libration observations can be used to prove the existence of these liquid layers and to constrain the thickness of the overlying solid layers. For Io, the presence of a small liquid core increases the libration of the mantle by a few percent with respect to an entirely solid Io and mantle libration observations could be used to determine the mantle thickness with a precision of several tens of kilometers given that the libration amplitude can be measured with a precision of one meter. For Europa, Ganymede, and Callisto, the presence of a water ocean close to the surface increases by at least an order of magnitude the ice shell libration amplitude with respect to an entirely solid satellite. The shell libration depends essentially on the shell thickness and to a minor extent on the density difference between the ocean and the ice shell. The possible presence of a liquid core inside Europa and Ganymede has no noticeable influence on their shell libration. For a precision of several meters on the libration measurements, in agreement with the expected accuracy with the NASA/ESA EJSM orbiter mission to Europa and Ganymede, an error on the shell thickness of a few tens kilometers is expected. Therefore, libration measurements can be used to detect liquid layers such as Io's core or water subsurface oceans in Europa, Ganymede, and Callisto and to constrain the thickness of the overlying solid surface layers.

Key words: Jupiter satellites, interiors, rotational dynamics, ice 


\section{Introduction}

Through the determination of the second-degree gravity field, image acquisition and magnetic field measurement, the Galileo mission provided information on the interior structure of the Galilean satellites Io, Europa, Ganymede, and Callisto (Anderson et al. 1996, 1998, 2001a and 2001b). Each satellite is thought to have one or two global internal liquid layers. In particular, Europa, Ganymede, and Callisto likely have an ocean beneath an overlying ice shell, while Io most likely has a fully liquid core beneath a silicate mantle. Europa and Ganymede could have a fluid metallic core as a second global internal liquid layer. Several methods, such as measurements of tidal gravity variations and tidal surface displacements and the observation of the magnetic field in the neighborhood of the satellite, have been proposed to assess the existence and the depth of the putative ocean beneath the ice surface in the particular case of Europa (e.g. Wahr et al. 2006 and Khurana et al. 1998). Also measurements of the librations (rotation variations) of Europa have been proposed as a means to charaterize the ice shell and ocean (Van Hoolst et al. 2008). Here we study the librations of the Galilean satellites and investigate whether they can be used to prove the existence of liquid layers and to constrain the thickness of the ice shell of Europa, Ganymede, and Callisto and of Io's mantle. The knowledge of this thicknesses is important for our understanding of the formation and the evolution of these satellites and for the study of their habitability.

Longitudinal librations are due to the gravitational attraction of Jupiter on the static equatorial bulges of the synchronously rotating Galilean satellites. Since the Galilean satellites orbits are eccentric, the orbital speed is vari- 
able and the static equatorial bulges are not always exactly oriented towards Jupiter. Therefore Jupiter exerts a time-variable gravitational torque on the satellites, modifying their rotation rate (e.g. Comstock and Bills 2003). For a satellite having one or two liquid layers, Jupiter's torque on the different internal layers must be considered and the librations are also modified by the internal gravitational, pressure, viscous and electromagnetic torques between their layers. The effect of a subsurface ocean on the librations of Europa and Titan has been addressed in Van Hoolst et al. (2008, 2009). Here we develop a more general framework for the study of librations of satellites in 1:1 resonance with up to two global internal liquid layers and assess expected improvements on the interior structure of the four Galilean satellites from future librations observations.

The plan of the paper is as follows. In the second section, we derive the governing equations for the libration of an entirely solid satellite and for a generic satellite with up to two global internal liquid layers by considering the changes in angular momentum of the layers resulting from gravitational coupling with Jupiter and torques between the different internal layers. Expressions for the internal and external torques on the layers of the generic satellites are determined in Section 3. In the fourth section, we solve the libration equations for each Galilean satellite for different possible internal structures and assess the influence of internal structure parameters on the libration amplitude. The last section presents a discussion and conclusion. 


\section{Angular momentum equations}

\subsection{Introduction}

The rotation of a satellite is described in an inertial reference frame. The orbital period of each Galilean satellite is shorter than the precession period of its orbit (e.g. Bills 2005) and shorter than the period of all other significant perturbations of their orbit (Lainey et al. 2004a and 2004b). Therefore, the orbital reference frame can be considered as an inertial frame for the librations at orbital period considered here. It will be denoted $x y z$, with the $z$-axis in the orbit normal direction and the $x$-axis taken to be the major axis of the orbit (Fig 1).

For the four Galilean satellites, the obliquity, which is the angle between the rotation axis and the orbit normal, is of the order of magnitude of the obliquity of the Cassini state (Bills 2005). These obliquities are at most of the order of a few degreees, suggesting that the obliquity can be neglected in the calculation of longitudinal libration. This approximation is correct even up to the first order in obliquity since the obliquity is of second order in the equation for longitudinal libration. To show that, one can use a similar reasoning as Peale (1988) has adopted for Mercury, which is in a 3:2 spin-orbit resonance.

We also neglect the wobble of the Galilean satellites. In these approximations, the inertial z-axis, the orbit normal, the rotation axis and the polar axis are then all in the same direction and the orientation in space of the rotation axis is constant. Therefore, we only have to consider the variations in rotation rate of the satellite (or longitudinal librations) and the angular momentum 
equations has only a z-component.

\subsection{Rigid libration}

For an entirely solid and rigid satellite, the z-component of the angular momentum equation can generally be expressed as

$$
C \ddot{\phi}=\Gamma
$$

where $C$ is the polar moment of inertia of the satellite, $\phi$ is the orientation angle (or rotation angle) between the long axis of the satellite (associated with the smallest moment of inertia $A$ ) and a direction fixed in space (here taken to be the major axis of the orbit), and $\Gamma$ is the $z$-component of the external torque on the satellite, which is dominated by Jupiter's gravitational torque. The torque is given by (see e.g. Murray and Dermott, 1999)

$$
\Gamma=\frac{3}{2}(B-A) \frac{G M_{\mathrm{J}}}{d^{3}} \sin 2 \psi
$$

and depends on the difference of the equatorial moments of inertia $(A<B)$ of the satellite, on the universal gravitational constant $G$, on the mass of Jupiter $M_{\mathrm{J}}$, on the distance $d$ between the mass centers of the satellite and Jupiter and on the angle $\psi$ between the axis of mimimum moment of inertia of the satellite and the direction from the satellite to Jupiter $(\psi=f-\phi$, where $f$ is the true anomaly). By neglecting the satellite's mass with respect to Jupiter's mass in Kepler's third law, $G M_{\mathrm{J}}=n^{2} a^{3}$, with $a$ the semi-major axis of the satellite's orbit, and $n$ the mean motion, we have

$$
\Gamma=K_{\text {grav }}\left(\frac{a}{d}\right)^{3} \sin 2 \psi
$$


where

$$
K_{\text {grav }}=\frac{3}{2}(B-A) n^{2}
$$

is the strength of the gravitational coupling.

The rigid rotational response is a good approximation for an entirely solid satellite without liquid layers. The solidity ensures that there will be no relative rotation of the different internal layers and that the elastic deformation effect is small. Deformation has an effect on libration by changing the polar moment of inertia of the satellite and hence also the angular momentum $H=(C+$ $\delta C(t)) \dot{\phi}$, where $\delta C(t)$ is the time-variable part of the polar moment of inertia. If we write $\dot{\phi}=\omega+\dot{\gamma}(t)$, with the libration angle $\gamma=\phi-M$ where $M$ is the mean anomaly of the satellite, and neglect the product of small quantities $\dot{\gamma}(t) \delta C(t)$, the angular momentum equation is then given by

$$
C \ddot{\phi}+\omega \frac{d \delta C(t)}{d t}=\Gamma
$$

and differs from Eq. (1) by an additional term in the left-hand member.

The deformation is a result of the libration itself as well as of the periodic tides. The changes in the rotation rate and in the distance between the planet and the satellite lead to changes in the centrifugal and tidal potentials, respectively, and therefore to a time-variable polar flattening. The tides also cause the equatorial flattening to vary in time, however, in the absence of dissipation processes, the periodic equatorial flattening is always aligned with the planetsatellite direction, and does not lead to a change in rotation. Even if we were to include tidal dissipation, the smallness of both the periodic equatorial flattening and its misalignment suggests that this contribution to the libration 
equation can be neglected, as has been shown for Enceladus (Rambaux et al. 2010).

The time-variable part of the polar moment of inertia due to the changing centrifugal potential as a result of libration can be expressed as (see e.g. Moritz and Mueller 1987)

$$
\delta C=k_{2} \frac{4 R^{5} \omega}{9 G}(\dot{\phi}-\omega)
$$

where $R$ is the mean radius, $\omega$ is the mean rotation rate ( $\omega=n$ for the Galilean satellites), and $k_{2}$ is the degree-two potential Love number of the satellite. The tidal time-variable part of the polar moment of inertia can be expressed as (see Van Hoolst et al. 2008)

$$
\delta C=k_{2} q \text { e } M_{\text {sat }} R^{2} \cos M
$$

where $M_{\text {sat }}$ is the mass of the satellite, $M$ is its mean anomaly, and $q=$ $n^{2} R^{3} / G M_{\text {sat }}$. The z-component of the angular momentum equation can then be expressed as

$$
C \ddot{\phi}+\omega k_{2} \frac{4 R^{5} \omega}{9 G} \ddot{\phi}-n^{2} k_{2} q e M_{s} R^{2} \sin M=\frac{3}{2}(B-A) \frac{G M_{\mathrm{J}}}{d^{3}} \sin 2 \psi
$$

The largest estimated values of $k_{2}$ for Io, Europa, Ganymede, and Callisto are $0.8,0.25,0.6$ and 0.5 , respectively. They correspond to models with a lowyiscosity astenosphere for Io (Lainey et al. 2009) and to models with a subsurface ocean and low ice shell rigidity $\left(\mu=10^{9} \mathrm{~Pa}\right)$ for Europa (Moore and Schubert, 2000 and Wahr et al. 2006), Ganymede, and Callisto (Moore and Schubert, 2003). If we assume the satellites to be entirely solid, the $k_{2}$ values will be about ten times lower. In Eq. 8, the term related to the librational deformation is more than a factor 500 smaller than the main term $C \ddot{\phi}$, even 
with the largest values for $k_{2}$, and will therefore be neglected in this study. The ratio of the term related to the tidal deformation and the gravitational torque is equal to 0.10, 0.04, 0.12 and 0.08 for Io, Europa, Ganymede, and Callisto, respectively, with the largest values of $k_{2}$ given above. This can be easily seen by using an expansion for the torque in the small eccentricity (Van Hoolst et al. 2008, or see Eq. 18). Therefore the effect of zonal tides on libration could be up to $\sim 10 \%$. Although this shows that the effect of zonal tides must be included when analysing measurements of rotation variations of the Galilean satellites, the Love numbers, and therefore the relative effect of zonal tides on libration, are an order of magnitude smaller for the solid satellites considered here. Therefore, and also because the Love numbers are not well known, tidal deformation will be neglected. This situation is different for the Earth, for which the librations are very small because the Earth is not in a 1:1 resonance (Chao et al. 1991).

Besides forced librations, Eq. (1) leads to a free libration with natural frequency $\sigma_{f}$. By averaging Eq. (1) over an orbital period, the free frequency $\sigma_{f}$ can be obtained as (e.g. Comstock and Bills 2003)

$$
\sigma_{f}=n \sqrt{\frac{3(B-A)}{C}} .
$$

For all Galilean satellites, it is much smaller than the mean motion $n$ since $B-A$ is much smaller than $C$ (the ratio $(B-A) / C$ is of the order of $C_{22}$, which has been determined from Galileo flybys to be of the order of $10^{-5}$, Anderson et al. 1996, 1998, 2001a and 2001b, see also Table B.1). Dissipation processes due to tides and libration damp the long-periodic free libration and its amplitude can be assumed to be zero. 
The forced librations have periods equal to the periods of the Jupiter torque. The main libration term has a frequency $n$ (period corresponding to the orbital period) and is therefore short-periodic with respect to the free libration (Comstock and Bills, 2003). We do not consider the much smaller forced librations with sub-harmonic periods here. For small librations, the amplitude of the forced libration can be approximated up to first order in eccentricity $e$ by (e.g. Murray and Dermott 2000)

$$
A_{\theta}=-6 \frac{(B-A)}{C} \frac{n^{2} e}{n^{2}-\sigma_{f}^{2}} \approx-6 \frac{(B-A)}{C} e
$$

The principal moments of inertia are the only interior structure parameters involved in the amplitude of the rigid libration of the Galilean satellites, and Eq. (10) shows that the libration amplitude is proportional to $(B-A) / C$. The proportionality to the moment of inertia difference $B-A$ is related to the proportionality of the gravitational torque to that difference and since $C$ is a measure of the resistance to a rotational motion, the libration is inversely proportional to the polar moment of inertia.

From the second-degree gravitational gravity field estimations of Anderson et al. $(1996,1998,2001 \mathrm{a}$ and $2001 \mathrm{~b})$, we evaluate the factor $(B-A) / C$ for the four satellites. The equatorial moment of inertia difference $(B-A)$ is given by $4 M R^{2} C_{22}$ while the polar moment of inertia $C$ can be approximated by the mean moment of inertia $I$ given by the radau equation or calculated by solving the Clairaut equation (Van Hoolst et al. 2008). The amplitude of the surface displacement in longitude at the equator due to rigid libration, given by $A_{\theta} R$, is then 269, 134, 10 and $12 \mathrm{~m}$ for Io, Europa, Ganymede, and Callisto, respectively (see Table B.1 for the values of $R, C_{22}$ and $I$ ). 
2.3 Librations of a generic satellite consisting of two global liquid and three solid layers

We define a generic satellite as made up of six homogenous layers (Fig. 2). From the surface to the center, we have: an ice shell $(s)$, a water ocean $(o)$, an ice mantle $(i)$, a rock mantle $(r)$, a fluid core $(f)$ and a solid core $(c)$. The solid layer composed of the ice and rock mantles will also be called mantle $(m)$.

When a subsurface ocean and/or a fluid core exists, the solid layers (shell, mantle and solid core) and the fluid layers (ocean and fluid core) can perform different rotation variations. However, they are coupled with each other through viscous, electromagnetic, pressure and gravitational torques. The viscous torques due to friction forces on the boundaries of the internal ocean and liquid core depend on the small ocean and liquid core viscosity which for both is of the order of a few mPas (Vočadlo, 2007). Therefore, the Ekman timescale is about 30000 years, much longer than the libration period of a few days. As a result, viscous torques cannot effectively couple solid to liquid layers at the libration periods and we hereafter neglect viscosity in the liquid layers. For a similar problem about the libration of Mercury, see e.g. Peale et al.(2002). For similar reasons we neglect the electromagnetic torque, although the electromagnetic field generated in the core of Ganymede could be large. The coupling between the five internal regions considered is then due to the gravitational force between layers and the pressure force of the liquid layers on their boundaries.

The $z$-component of the angular momentum equations for the shell, ocean, 
mantle, fluid core and solid inner core of the satellite can be written as

$$
\begin{aligned}
C_{s} \ddot{\phi}_{s} & =\Gamma_{s}, \\
C_{o} \dot{\omega}_{o} & =\Gamma_{o}, \\
C_{m} \ddot{\phi}_{m} & =\Gamma_{m}, \\
C_{f} \dot{\omega}_{f} & =\Gamma_{f}, \\
C_{c} \ddot{\phi}_{c} & =\Gamma_{c} .
\end{aligned}
$$

Here, $C_{j}$ and $\phi_{j}$ are the polar principal moment of inertia and the rotation angle of layer $j$, and $\Gamma_{j}$ is the total torque on layer $j$. In contrast to the solid layers, the changes in angular momentum of the ocean and of the fluid core are written in terms of the rotation frequencies $\omega_{0}$ and $\omega_{f}$, defined in such a way that $C_{o} \omega_{o}$ and $C_{f} \omega_{f}$ are equal to the angular momentum of the ocean and of the fluid core. In principle, Eqs. (11)-(15) should also include terms related to the librational and tidal deformations of the layers, but as for the entirely solid satellite, we neglect the elastic response, and consider the solid layers to be rigid. Other types of deformation can also occur when a liquid layer exists, due to gravitational interaction between layers that are misaligned. However, these deformations are expected to be smaller than the neglected tidal deformation from a comparison of the potentials causing these deformations. For example, we find the potential related to misalignment of the solid interior with respect to the ice shell to be an order of magnitude smaller than the tidal potential for the libration amplitudes obtained in this study.

\section{Torques}

The z-components of the total torques on the different layers are due to gravitational interaction with Jupiter, to internal gravitational forces between the 
internal layers of the satellite, and to pressure forces on the boundaries of the region considered. Internal gravitational coupling arises when the principal axes of two layers are not aligned. Since we only study the rotation about the $z$-axis, we consider differences in the orientation of the smallest moment of inertia axis $A_{j}$ of layers $j$. The $A$-axis of a solid layer is given by the long axis of the ellipsoidal top and bottom surfaces of the layer. For a liquid layer, the orientation of the long axis changes because of orientation changes of the top and bottom boundaries with the solid neighboring layers (see Fig. 3). Misalignment of the boundaries occurs when Jupiter's gravitational torque forces the solid layers to change their rotation at a different rate. Upon differential rotation, the fluid layers will also exert pressure torques on the solid layers.

We write the torque on an internal layer $j$ as

$$
\Gamma_{j}=\Gamma_{J, j}+\Gamma_{g, j}+\Gamma_{p, j}
$$

where $\Gamma_{J, j}$ is Jupiter's gravitational torque on layer $j, \Gamma_{g, j}$ is the internal gravitational torque on layer $j$, and $\Gamma_{p, j}$ is the pressure torque on layer $j$.

\subsection{Jupiter's gravitational torque}

The gravitational torque of Jupiter on the internal layers can be expressed similarly as Eq. (2) for the torque on the total satellite. We have

$$
\Gamma_{J, j}=\frac{3}{2}\left(B_{j}-A_{j}\right) \frac{G M_{\mathrm{J}}}{d^{3}} \sin 2 \psi_{j}
$$

where $\psi_{j}$ is the angle between the long axis of layer $j$ (associated with the smallest moment of inertia $A_{j}$ of layer $j$ ) and the direction from the satellite to Jupiter $\left(\psi_{j}=f-\phi_{j}\right)$. Up to the first order in the small eccentricity and 
libration angle $\gamma_{j}=\phi_{j}-M$, this can be approximated by

$$
\Gamma_{J, j}=6 e n^{2}\left(B_{j}-A_{j}\right) \sin M-3 n^{2}\left(B_{j}-A_{j}\right) \gamma_{j}
$$

(e.g. Van Hoolst et al. 2008).

For a solid layer, the angles $\phi_{j}$ and $\gamma_{j}$ are determined by the long axis of their surfaces. Since Jupiter's gravitational torque on a fluid layer depends on the orientation of the two surrounding solid layers, we re-express the right-hand side of Eq. (17) for the fluid layers in terms of the orientation angles of the two surrounding solid layers. To do so, we divide the fluid layer into two regions: a bottom part below an arbitrarily chosen sphere inside the fluid layer and a top part above that sphere. The gravitational torque on the fluid layer can then be expressed as the sum of the torques on the aspherical bottom and top parts of the fluid layers, denoted by $b$ and $t$. We have

$$
\begin{aligned}
\Gamma_{J, o} & =\frac{3}{2} \frac{G M_{\mathrm{S}}}{d^{3}}\left[\left(B_{o, b}-A_{o, b}\right) \sin 2 \psi_{m}+\left(B_{o, t}-A_{o, t}\right) \sin 2 \psi_{s}\right] \\
\Gamma_{J, f} & =\frac{3}{2} \frac{G M_{\mathrm{S}}}{d^{3}}\left[\left(B_{f, b}-A_{f, b}\right) \sin 2 \psi_{c}+\left(B_{f, t}-A_{f, t}\right) \sin 2 \psi_{m}\right] .
\end{aligned}
$$

The equatorial moment of inertia difference of an internal layer $B_{\text {int }}-A_{\text {int }}$ between mean radii $r_{1}<r_{2}$ can be expressed, correct up to the first order in the flattening, as

$$
B_{\mathrm{int}}-A_{\mathrm{int}}=\frac{8 \pi}{15} \int_{r_{1}}^{r_{2}} \rho\left(r_{0}^{\prime}\right) \frac{d\left(\beta r_{0}^{\prime 5}\right)}{d r_{0}^{\prime}} d r_{0}^{\prime}
$$

Here, $\rho\left(r_{0}\right)$ is the density of a spherical reference model at radius $r_{0}$ and $\beta\left(r_{0}\right)$ the equatorial flattening of an internal layer with mean radius $r_{0}$. The equatorial flattening is defined as the relative difference $(a-b) / a$ of the longest and smallest radii of the surface in the equator plane. For example, for the 
top and bottom layers of the ocean and of the fluid core, which we assume to have a uniform density, we then have

$$
\begin{aligned}
B_{o, b}-A_{o, b} & =-\frac{8 \pi}{15} \rho_{o} \beta_{m} R_{m}^{5} \\
B_{o, t}-A_{o, t} & =\frac{8 \pi}{15} \rho_{o} \beta_{o} R_{o}^{5} \\
B_{f, b}-A_{f, b} & =-\frac{8 \pi}{15} \rho_{f} \beta_{c} R_{c}^{5} \\
B_{f, t}-A_{f, t} & =\frac{8 \pi}{15} \rho_{f} \beta_{f} R_{f}^{5}
\end{aligned}
$$

where $\rho_{o}$ and $\rho_{f}$ are the density of the ocean and of the fluid core, $R_{m}, R_{o}, R_{c}$ and $R_{f}$ are the mean radius of the mantle, of the ocean, of the solid core and of the fluid core, $\beta_{m}, \beta_{o}, \beta_{c}$ and $\beta_{f}$ are the equatorial flattening of the mantle, of the ocean, of the solid core and of the fluid core. The signs of $B_{o, b}-A_{o, b}$ and $B_{f, b}-A_{f, b}$ are different of those of $B_{o, t}-A_{o, t}$ and $B_{f, t}-A_{f, t}$ because for the bottom ocean and the bottom fluid core, the outer surface is spherical and the inner surface is ellipsoidal, while the situation is reversed for the top ocean and the top fluid core.

\subsection{Internal gravitational torques}

When two layers are not aligned, their gravitational fields do not have the same geometry, and the layers will exert a gravitational torque on each other. The internal gravitational torque on layer $j$ can be expressed as

$$
\Gamma_{g, j}=-\int_{V_{j}}\left(\vec{r} \wedge \rho_{j} \nabla \Phi\right)_{z} d V
$$

where $\Phi$ is the gravitational potential due to the internal masses of the satellite and $V_{j}$ the volume of layer $j$. Obviously, the masses of the layer $j$ itself do not contribute to the torque and only the masses located outside layer $j$ need 
to be taken into account. In App. A.1 and A.2, we derive expressions for the gravitational torque on layer $j$ due to another layer of the satellite lying closer to or further away from the mass center than layer $j$. Here, we apply these expressions to obtain the gravitational torque on each of the three solid and two liquid layers.

The torque on the solid core is due entirely to layers located further away from the center and only two orientations besides the orientation of the solid core are needed to describe the gravitational coupling. First, the mantle and both the top part of the fluid core and the bottom part of the ocean, which both are aligned with the mantle, exert a torque on the solid core. Second, the masses inside the shell and the part of the ocean aligned with the shell contribute to the torque. Therefore, the internal gravitational torque on the solid core is given by the sum of Eq. A.19 applied to these two masses. We have

$$
\begin{aligned}
\Gamma_{g, c}= & \frac{4 \pi G}{5}\left[B_{c}-A_{c}\right]\left[\rho_{f} \beta_{f}+\rho_{r}\left(\beta_{r}-\beta_{f}\right)+\rho_{i}\left(\beta_{i}-\beta_{r}\right)+\rho_{o}\left(-\beta_{i}\right)\right] \\
& \times \sin 2\left(\phi_{m}-\phi_{c}\right) \\
& +\frac{4 \pi G}{5}\left[B_{c}-A_{c}\right]\left[\rho_{o} \beta_{o}+\rho_{s}\left(\beta_{s}-\beta_{o}\right)\right] \sin 2\left(\phi_{s}-\phi_{c}\right) .
\end{aligned}
$$

The torque on the mantle is due, firstly, to the shell and the part of the ocean aligned with the shell (masses located further away from the center than the mantle) and, secondly, to the solid core and the bottom part of the fluid core aligned with the solid core (masses located closer to the center). Equation A.19 can be used for the first contribution and Eq. A.21 applies to the second. Addition of the two contributions gives 


$$
\begin{aligned}
\Gamma_{g, m}= & \frac{4 \pi G}{5}\left(B_{m}-A_{m}\right)\left[\rho_{o} \beta_{o}+\rho_{s}\left(\beta_{s}-\beta_{o}\right)\right] \sin 2\left(\phi_{s}-\phi_{m}\right) \\
& -\frac{4 \pi G}{5}\left[\left(B_{c}-A_{c}\right)+\left(B_{f, b}-A_{f, b}\right)\right]\left[\rho_{r}\left(\beta_{r}-\beta_{f}\right)+\rho_{i}\left(\beta_{i}-\beta_{r}\right)\right] \\
& \times \sin 2\left(\phi_{m}-\phi_{c}\right) .
\end{aligned}
$$

The torque on the shell is due to masses located closer to the center than the shell: the solid core and the bottom part of the fluid core aligned with it, and the mantle and the parts of the fluid layers aligned with it. Application of Eq. A.21 then leads to

$$
\begin{aligned}
\Gamma_{g, s}= & -\frac{4 \pi G}{5}\left[\left(B_{c}-A_{c}\right)+\left(B_{f, b}-A_{f, b}\right)\right]\left[\rho_{s}\left(\beta_{s}-\beta_{o}\right)\right] \sin 2\left(\phi_{s}-\phi_{c}\right) \\
& -\frac{4 \pi G}{5}\left[\left(B_{m}-A_{m}\right)+\left(B_{f, t}-A_{f, t}\right)+\left(B_{o, b}-A_{o, b}\right)\right] \\
\times & {\left[\rho_{s}\left(\beta_{s}-\beta_{o}\right)\right] \sin 2\left(\phi_{s}-\phi_{m}\right) . }
\end{aligned}
$$

The torques on the fluid layers are somewhat more complicated to derive than the torques on the solid layers, because the top and bottom parts of the liquid layers can be differently oriented. Therefore, we have to calculate the torque on the bottom part and on the top part separately and sum the results afterwards. We have

$$
\begin{aligned}
\Gamma_{g, o}= & \frac{4 \pi G}{5}\left(B_{o, b}-A_{o, b}\right)\left[\rho_{s}\left(\beta_{s}-\beta_{o}\right)\right] \sin 2\left(\phi_{s}-\phi_{m}\right) \\
& -\frac{4 \pi G}{5}\left[\left(B_{c}-A_{c}\right)+\left(B_{f, b}-A_{f, b}\right)\right]\left[-\rho_{o} \beta_{i}\right] \sin 2\left(\phi_{m}-\phi_{c}\right) \\
& -\frac{4 \pi G}{5}\left[\left(B_{m}-A_{m}\right)+\left(B_{f, t}-A_{f, t}\right)\right]\left[\rho_{o} \beta_{o}\right] \sin 2\left(\phi_{s}-\phi_{m}\right) \\
& -\frac{4 \pi G}{5}\left[\left(B_{c}-A_{c}\right)+\left(B_{f, b}-A_{f, b}\right)\right]\left[\rho_{o} \beta_{o}\right] \sin 2\left(\phi_{s}-\phi_{c}\right),
\end{aligned}
$$




$$
\begin{aligned}
\Gamma_{g, f}= & \frac{4 \pi G}{5}\left(B_{f, b}-A_{f, b}\right)\left[\rho_{r}\left(\beta_{r}-\beta_{f}\right)+\rho_{i}\left(\beta_{i}-\beta_{r}\right)-\rho_{o} \beta_{i}\right] \\
& \times \sin 2\left(\phi_{m}-\phi_{c}\right) \\
& +\frac{4 \pi G}{5}\left(B_{f, b}-A_{f, b}\right)\left[\rho_{o} \beta_{o}+\rho_{s}\left(\beta_{s}-\beta_{o}\right)\right] \sin 2\left(\phi_{s}-\phi_{c}\right) \\
& -\frac{4 \pi G}{5}\left(B_{c}-A_{c}\right)\left[\rho_{f} \beta_{f}\right] \sin 2\left(\phi_{m}-\phi_{c}\right) \\
& +\frac{4 \pi G}{5}\left(B_{f, t}-A_{f, t}\right)\left[\rho_{o} \beta_{o}+\rho_{s}\left(\beta_{s}-\beta_{o}\right)\right] \sin 2\left(\phi_{s}-\phi_{m}\right) .
\end{aligned}
$$

\subsection{Internal pressure torques}

The pressure torque on layer $j$ is given by

$$
\Gamma_{p, j}=-\int_{S_{j}}\left(\vec{r}_{j} \wedge \hat{n}_{j}\right)_{z} P d S+\int_{S_{j-1}}\left(\vec{r}_{j-1} \wedge \hat{n}_{j-1}\right)_{z} P d S
$$

where $S_{j}$ and $S_{j-1}$ are the top and bottom surfaces of the layer $j$, and $\hat{n}_{j}$ and $\hat{n}_{j-1}$ are the outward unit normals on $S_{j}$ and $S_{j-1}$. The pressure torques on the shell and on the solid core are particular cases of the previous equation because the pressure is zero at the top surface of the shell (considering there is no atmosphere) and the solid core has no bottom surface.

$$
\begin{aligned}
\Gamma_{p, s} & =\int_{S_{o}}\left(\vec{r}_{o} \wedge \hat{n}_{o}\right)_{z} P d S, \\
\Gamma_{p, c} & =-\int_{S_{c}}\left(\vec{r}_{c} \wedge \hat{n}_{c}\right)_{z} P d S .
\end{aligned}
$$

To calculate the pressure in a liquid layer, the flow in the liquid must be studied. This flow can be quite complicated (e.g. Noir et al. 2009), but we here only consider the flow that can contribute to the rotation variations of the solid layers. For an incompressible, homogeneous liquid inside a solid ellipsoidal cavity, Hough (1895) proved that such a flow is a uniform vorticity flow together with a potential flow, which is a factor of the order of the flat- 
tening of the cavity smaller than the rigid body rotation and is required to satisfy the boundary conditions. This 'Poincaré' flow (Poincaré 1910) has been shown to be a good approximation in more complicated situations, notably it has been demonstrated that for the rotation variations of the Earth, the flow in the Earth's outer fluid core is of the form of the Poincaré flow, correct up to the first order in the flattening (Mathews et al. 1991). Since the equilibrium rotation is around the z-axis, the Poincaré flow does not contribute to the z-component of torque on the solid layers in the first-order approximation in the small rotation variations and the z-component of the pressure torque can be calculated from the hydrostatic balance (see, e.g. Hinderer et al. 1982, Mathews et al. 1991, Dumberry 2008, Van Hoolst et al. 2009)

$$
\nabla P=-\rho \nabla \Phi-\rho \nabla W
$$

with $\rho$ the density of the fluid considered. The contribution of the external gravitational potential to the fluid pressure was not considered in the study of the librations of Europa by Van Hoolst et al. (2008).

The pressure can be decomposed into a part related to the internal satellite potential $\Phi$ and a part due to the external potential of Jupiter $W$ :

$$
\begin{aligned}
& \nabla P_{g}=-\rho \nabla \Phi, \\
& \nabla P_{J}=-\rho \nabla W,
\end{aligned}
$$

and the pressure torque can likewise be decomposed into two parts:

$$
\Gamma_{p, j}=\Gamma_{p g, j}+\Gamma_{p J, j}
$$

In Appendix B, we derive expressions for both parts of the pressure torque. Application of Eqs. (B.6) and (B.12) gives the following results for the pressure 
torques related to the satellite potential on the solid core, mantle and shell:

$$
\begin{aligned}
\Gamma_{p g, c}= & \frac{4 \pi G}{5}\left[\rho_{f} \beta_{f}+\rho_{r}\left(\beta_{r}-\beta_{f}\right)+\rho_{i}\left(\beta_{i}-\beta_{r}\right)+\rho_{o}\left(-\beta_{i}\right)\right] \\
& \times\left[\left(B_{f, b}-A_{f, b}\right)\right] \sin 2\left(\phi_{m}-\phi_{c}\right) \\
& +\frac{4 \pi G}{5}\left[\left(B_{f, b}-A_{f, b}\right)\right]\left[\rho_{o} \beta_{o}+\rho_{s}\left(\beta_{s}-\beta_{o}\right)\right] \sin 2\left(\phi_{s}-\phi_{c}\right), \\
\Gamma_{p g, m}= & \frac{4 \pi G}{5}\left[\left(B_{f, t}-A_{f, t}\right)+\left(B_{o, b}-A_{o, b}\right)\right]\left[\rho_{o} \beta_{o}+\rho_{s}\left(\beta_{s}-\beta_{o}\right)\right] \\
& \times \sin 2\left(\phi_{s}-\phi_{m}\right) \\
& -\frac{4 \pi G}{5}\left[\left(B_{c}-A_{c}\right)+\left(B_{f, b}-A_{f, b}\right)\right]\left[\beta_{f} \rho_{f}-\beta_{i} \rho_{o}\right] \\
& \times \sin 2\left(\phi_{m}-\phi_{c}\right), \\
\Gamma_{p g, s}= & -\frac{4 \pi G}{5}\left[\left(B_{c}-A_{c}\right)+\left(B_{f, b}-A_{f, b}\right)\right]\left[\rho_{o} \beta_{o}\right] \sin 2\left(\phi_{s}-\phi_{c}\right) \\
& -\frac{4 \pi G}{5}\left[\left(B_{m}-A_{m}\right)+\left(B_{f, t}-A_{f, t}\right)+\left(B_{o, b}-A_{o, b}\right)\right]\left[\rho_{o} \beta_{o}\right] \\
& \times \sin 2\left(\phi_{s}-\phi_{m}\right) .
\end{aligned}
$$

For the torques on the liquid parts, we obtain the interesting result that the pressure torque related to the internal gravitational potential fully compensates the internal gravitational torque: $\Gamma_{p g, f}=-\Gamma_{g, f}$ and $\Gamma_{p g, o}=-\Gamma_{g, o}$. For a similar result for the torque on the outer core of the Earth, see e.g. Hinderer et al. (1982).

For the pressure torques related to the Jupiter potential, we have, using (B.18)

$$
\begin{aligned}
\Gamma_{p J, c} & =\frac{3}{2}\left(B_{f, b}-A_{f, b}\right) \frac{G M_{p}}{d^{3}} \sin 2 \psi_{c}, \\
\Gamma_{p J, m} & =\frac{3}{2}\left[\left(B_{f, t}-A_{f, t}\right)+\left(B_{o, b}-A_{o, b}\right)\right] \frac{G M_{p}}{d^{3}} \sin 2 \psi_{m}, \\
\Gamma_{p J, s} & =\frac{3}{2}\left(B_{o, t}-A_{o, t}\right) \frac{G M_{p}}{d^{3}} \sin 2 \psi_{s} .
\end{aligned}
$$

For the liquid parts, we have $\Gamma_{p J, f}=-\Gamma_{J, f}$ and $\Gamma_{p J, o}=-\Gamma_{J, o}$. Therefore, the total torques on the ocean and on the fluid core $\Gamma_{o}$ and $\Gamma_{f}$ are zero.

From the angular momentum equations for the ocean (12) and for the liquid 
core (14), we then have, in the Poincaré approximation $\left(\dot{h}_{o}\right.$ and $\left.\dot{h}_{f} \simeq 0\right)$,

$$
\begin{aligned}
C_{o} \dot{\omega}_{o} & =0, \\
C_{f} \dot{\omega}_{f} & =0 .
\end{aligned}
$$

It follows that the ocean and the fluid core do not take part in the libration of the satellite and rotate at constant rotation frequency $n$.

\section{Results}

\subsection{Libration solutions}

The system of angular momentum Eqs. (11)-(15) can be reduced to three equations for the solid layers:

$$
\begin{aligned}
C_{s} \ddot{\phi}_{s} & =\Gamma_{g, s}+\Gamma_{J, s}+\Gamma_{p g, s}+\Gamma_{p J, s}, \\
C_{m} \ddot{\phi}_{m} & =\Gamma_{g, m}+\Gamma_{J, m}+\Gamma_{p g, m}+\Gamma_{p J, m}, \\
C_{c} \ddot{\phi}_{c} & =\Gamma_{g, c}+\Gamma_{J, c}+\Gamma_{p g, c}+\Gamma_{p J, c} .
\end{aligned}
$$

By using the torque expressions of Sec. 3 and expanding the distance to Jupiter and true anomaly as series in mean anomaly $M$ and eccentricity (see Eq. 18), we have correct up to the first order in the small libration angles $\gamma_{j}=\phi_{j}-M$ and eccentricity $e$

$$
\begin{aligned}
C_{s} \ddot{\gamma}_{s}+2 K_{s} \gamma_{s}+2 K_{s m}\left(\gamma_{s}-\gamma_{m}\right)+2 K_{s c}\left(\gamma_{s}-\gamma_{c}\right) & =4 e K_{s} \sin M \\
C_{m} \ddot{\gamma}_{m}+2 K_{m} \gamma_{m}-2 K_{s m}\left(\gamma_{s}-\gamma_{m}\right)+2 K_{m c}\left(\gamma_{m}-\gamma_{c}\right) & =4 e K_{m} \sin M \\
C_{c} \ddot{\gamma}_{c}+2 K_{c} \gamma_{c}-2 K_{m c}\left(\gamma_{m}-\gamma_{c}\right)-2 K_{s c}\left(\gamma_{s}-\gamma_{c}\right) & =4 e K_{c} \sin M
\end{aligned}
$$

with 


$$
\begin{aligned}
K_{s c}= & \frac{4 \pi G}{5}\left[\left(B_{c}-A_{c}\right)+\left(B_{f, b}-A_{f, b}\right)\right]\left[\rho_{o} \beta_{o}+\rho_{s}\left(\beta_{s}-\beta_{o}\right)\right] \\
K_{m c}= & \frac{4 \pi G}{5}\left[\left(B_{c}-A_{c}\right)+\left(B_{f, b}-A_{f, b}\right)\right] \\
& \times\left[\rho_{f} \beta_{f}+\rho_{r}\left(\beta_{r}-\beta_{f}\right)+\rho_{i}\left(\beta_{i}-\beta_{r}\right)+\rho_{o}\left(-\beta_{i}\right)\right] \\
K_{s m}= & \frac{4 \pi G}{5}\left[\left(B_{m}-A_{m}\right)+\left(B_{f, t}-A_{f, t}\right)+\left(B_{o, b}-A_{o, b}\right)\right] \\
& \times\left[\rho_{o} \beta_{o}+\rho_{s}\left(\beta_{s}-\beta_{o}\right)\right] \\
K_{s}= & \frac{3}{2} n^{2}\left[\left(B_{s}-A_{s}\right)+\left(B_{o, t}-A_{o, t}\right)\right] \\
K_{m}= & \frac{3}{2} n^{2}\left[\left(B_{m}-A_{m}\right)+\left(B_{f, t}-A_{f, t}\right)+\left(B_{o, b}-A_{o, b}\right)\right] \\
K_{c}= & \frac{3}{2} n^{2}\left[\left(B_{c}-A_{c}\right)+\left(B_{f, b}-A_{f, b}\right)\right] .
\end{aligned}
$$

Since we will consider the solid layers to be of homogenous density, we also use

$$
\begin{aligned}
\left(B_{c}-A_{c}\right) & =\frac{8 \pi}{15} \rho_{c} \beta_{c} R_{c}^{5} \\
\left(B_{m}-A_{m}\right) & =\frac{8 \pi}{15}\left[\rho_{i}\left(\beta_{i} R_{i}^{5}-\beta_{r} R_{r}^{5}\right)+\rho_{r}\left(\beta_{r} R_{r}^{5}-\beta_{f} R_{f}^{5}\right)\right], \\
\left(B_{s}-A_{s}\right) & =\frac{8 \pi}{15} \rho_{s}\left(\beta_{s} R^{5}-\beta_{o} R_{o}^{5}\right) .
\end{aligned}
$$

Equations (50-52) show that Jupiter's torque forces librations at orbital frequency $n$. We therefore seek solution of the form $\gamma_{s}=g_{s} \sin M, \gamma_{m}=g_{m} \sin M$ and $\gamma_{c}=g_{c} \sin M$. Librations at harmonic frequencies are not considered here since they are of higher order in the small eccentricity. We then have

$$
\begin{array}{r}
{\left[-C_{s} n^{2}+2\left(K_{s}+K_{s m}+K_{s c}\right)\right] g_{s}-2 K_{s m} g_{m}-2 K_{s c} g_{c}=4 e K_{s}} \\
-2 K_{s m} g_{s}+\left[-C_{m} n^{2}+2\left(K_{m}+K_{s m}+K_{m c}\right)\right] g_{m}-2 K_{m c} g_{c}=4 e K_{m} \\
-2 K_{s c} g_{s}-2 K_{m c} g_{m}+\left[-C_{c} n^{2}+2\left(K_{c}+K_{m c}+K_{s c}\right)\right] g_{m}=4 e K_{c}
\end{array}
$$

The libration amplitudes $g_{s}, g_{m}$ and $g_{c}$ can easily be calculated and can be expressed in the form 


$$
g_{j}=\frac{\chi_{j}}{\left(n^{2}-\sigma_{1}^{2}\right)\left(n^{2}-\sigma_{2}^{2}\right)\left(n^{2}-\sigma_{3}^{2}\right)},
$$

where $\sigma_{1}, \sigma_{2}$ and $\sigma_{3}$ are the eigenvalues of the system of Eqs. (50-52) with right-hand side set equal to zero. Because the expressions for the $\chi_{j}$ and eigenfrequencies are quite long, they are not written explicitely here. Explicit expressions in the case of one liquid layer are given in the next section.

For each satellite, we compute internal stucture models with homogenous layers of constant density consistent with their mean moment of inertia and mean density. We assume all satellites to be in hydrostatic equilibrium. For Io, the hydrostatic ratio $J_{2}=(10 / 3) C_{22}$ is well satisfied (Anderson et al. 2001b). The form of the equipotential surfaces, such as the boundaries of the layers, are assumed to be entirely due to rotation and static tides and therefore take the form of triaxial ellipsoids. By solving the Clairaut equation, we calculate the polar and equatorial flattening of each layer for all internal structure models (see Van Hoolst et al. 2008). These flattenings allow us to calculate $C_{j}$ and $\left(B_{j}-A_{j}\right)$. We compute the forced libration amplitude for the solid layers of the four Galilean satellites and assess the dependence of the amplitude on key interior stucture parameters.

\subsection{Ganymede}

Ganymede has a low mean moment of inertia which is compatible with a differentiated interior separated into an iron core, a rock mantle and an overlying outer ice layer (Anderson et al. 1996). Because Ganymede has a permanent magnetic dipole (Kivelson et al. 1998) for which dynamo action is the most likely explanation (Schubert et al. 2004), the core is likely to be separated 
into a solid inner core and a fluid outer core. The existence of an ocean inside the ice layer is still debated but possible detection of an induced magnetic field (Kivelson et al. 2002) provides a strong argument in favor of a subsurface ocean lying between an ice I shell and a deeper high pressure ice layer. We therefore here take as a reference model for Ganymede the same internal structure as that of the generic satellite, with two liquid layers (water ocean and fluid core).

The depth to the ocean is not accurately constrained. Kivelson et al. (2002) gave a shell thickness of the order of $150 \mathrm{~km}$, based on the analysis of the induced magnetic field. Here, we consider a range for the shell thickness, from 25 $\mathrm{km}$ up to $500 \mathrm{~km}$, in order to investigate more possible models and to highlight the link between the libration and the shell thickness. The density of the fluid core, $\rho_{f}$, is taken to be within the range $(5150-8000) \mathrm{kg} \mathrm{m}^{-3}$ (corresponding to an eutectic iron-sulfur mixture and pure iron, Anderson et al. 1996). The solid core is most likely composed of pure iron, with a density $\rho_{c}=8000 \mathrm{~kg}$ $\mathrm{m}^{-3}$, because at the core pressures in Ganymede sulfur partitions in the liquid. Nevertheless, because of our ignorance of the exact composition of the core, we consider also smaller values, up to $\rho_{c}=5150 \mathrm{~kg} \mathrm{~m}^{-3}$, corresponding to the density of the fluid core. The density of the rock mantle is taken to be within the range $(3000-3800) \mathrm{kg} \mathrm{m}^{-3}$ (from the lowest non-hydrated silicate density, Anderson et al. 1998, to the highest density of a silicate-metal mixture in Ganymede conditions, Anderson et al. 1996). The shell and the water ocean have a density $\rho_{s}$ and $\rho_{o}$ between 800 and $1200 \mathrm{~kg} \mathrm{~m}^{-3}$ to take into account the effect of possible impurities and porosity. The ice mantle consists of high pressure ice phases with density $\rho_{i}$ taken to be within the range $1000-1400$ $\mathrm{kg} \mathrm{m}^{-3}$. 
An infinity of internal structure models, determined by the values of the density and the radius of the six layers, is possible because we have only three data (total radius, mean density and mean moment of inertia) to constrain model parameters. We build a wide and representative range of plausible models by taking values for $\rho_{s}, \rho_{o}, \rho_{i}, \rho_{c}, R_{o}, R_{i}, R_{r}, R_{f}$ and $R_{c}$ from the appropriate ranges and by computing the values of $\rho_{r}$ and $\rho_{f}$ that satisfy the data (see Fig. 4 for an example of a density profile and Table B.2 for the values of the internal parameters). Our models respect the relations $\rho_{s} \leq \rho_{o} \leq \rho_{i}$ and $\rho_{f} \leq \rho_{c}$. Among our models, the water-ice layer has a maximum thickness of $\simeq 1150$ km, as in Anderson et al. 1996 and the ocean-ice shell layer has a maximum thickness of $\simeq 730 \mathrm{~km}$.

We calculated the forced libration amplitudes of the solid layers, for each model, from Eqs. (62)-(64) derived for the generic satellite. The forced libration amplitude of the shell at the equator, $R g_{s}$, is between 15 and $355 \mathrm{~m}$ (see Fig. 5). This is larger than the $\simeq 10 \mathrm{~m}$ libration amplitude of an entirely solid Ganymede. As can be seen from Fig. 5, the libration amplitude of the shell depends mainly on the shell thickness $h_{s}$. The amplitude also depends to a minor extent on the difference $\left(\rho_{o}-\rho_{s}\right)$, but only very weakly on all other interior structure parameters.

The amplitude of the libration of the mantle and the solid core, $R_{m} g_{m}$ and $R_{c} g_{c}$, are within the ranges $2-5 \mathrm{~m}$ and $0-0.5 \mathrm{~m}$, respectively. In principle, the core and mantle libration induce a time-dependent component in the seconddegree gravity coefficient $C_{22}$. The complete expression for $C_{22}$, with librations, is

$$
C_{22}=\frac{B-A}{4 M R^{2}}+\Delta C_{22}(t)=38.2610^{-6}+\Delta C_{22}(t),
$$


where

$$
\begin{aligned}
\Delta C_{22}= & \frac{\left(B_{s}-A_{s}\right)+\left(B_{o, t}-A_{o, t}\right)}{4 M R^{2}}\left(\cos 2 \gamma_{s}-1\right) \\
& +\frac{\left(B_{o, b}-A_{o, b}\right)+\left(B_{m}-A_{m}\right)+\left(B_{f, t}-A_{f, t}\right)}{4 M R^{2}}\left(\cos 2 \gamma_{m}-1\right) \\
& +\frac{\left(B_{f, b}-A_{f, b}\right)+\left(B_{c}-A_{c}\right)}{4 M R^{2}}\left(\cos 2 \gamma_{c}-1\right)
\end{aligned}
$$

The maximal individual effect of the shell libration on $C_{22}$ is reached for $\gamma_{s}=g_{s}$ and is given by $\left[\left(B_{s}-A_{s}\right)+\left(B_{o, t}-A_{o, t}\right)\right]\left(\cos 2 g_{s}-1\right) /\left(4 M R^{2}\right)$. At best, this effect is of the order of magnitude of $10^{-12}$ and is very small compared to the mean value of $C_{22}$. The effects of the mantle $\left(g_{m}\right)$ and of the core $\left(g_{c}\right)$ librations are below 3 and $0.002 \%$, respectively, of the effect of $g_{s}$ and are most likely too weak to be observed.

It is important to note that the libration amplitude of the shell almost does not depend on the size and density of the fluid core. This suggests that the shell libration is almost independent of whether the core is liquid or solid. We verify this statement by calculating the shell libration for an entirely solid core and we consider the limit case of Eqs. (62)-(64) for which the whole interior beneath the ocean is considered to be solid. Therefore, in the libration equations, we set $K_{c}, K_{m c}, K_{s c}$ and $C_{c}$ equal zero and take the mantle to extend to the center of the satellite. $K_{s m}$ is the only remaining internal coupling constant and will hereafter be denoted by $K_{\text {int }}$, as in Van Hoolst et al. (2008). Two libration equations (for the shell and the interior) are then needed, which can be expressed as

$$
\begin{aligned}
C_{s} \ddot{\gamma}_{s}+2 K_{s} \gamma_{s}+2 K_{i n t}\left(\gamma_{s}-\gamma_{i n}\right) & =4 e K_{s} \sin M, \\
C_{i n} \ddot{\gamma}_{i n}+2 K_{i n} \gamma_{i n}-2 K_{i n t}\left(\gamma_{s}-\gamma_{i n}\right) & =4 e K_{i n} \sin M,
\end{aligned}
$$

with 


$$
\begin{aligned}
K_{\text {int }}= & \frac{4 \pi G}{5}\left[\left(B_{i n}-A_{i n}\right)+\left(B_{o, b}-A_{o, b}\right)\right]\left[\rho_{o} \beta_{o}+\rho_{s}\left(\beta_{s}-\beta_{o}\right)\right], \\
K_{s}= & \frac{3}{2} n^{2}\left[\left(B_{s}-A_{s}\right)+\left(B_{o, t}-A_{o, t}\right)\right] \\
K_{i n}= & \frac{3}{2} n^{2}\left[\left(B_{i n}-A_{i n}\right)+\left(B_{o, b}-A_{o, b}\right)\right], \\
\left(B_{i n}-A_{i n}\right)= & \frac{8 \pi}{15}\left[\rho_{i}\left(\beta_{i} R_{i}^{5}-\beta_{r} R_{r}^{5}\right)+\rho_{r}\left(\beta_{r} R_{r}^{5}-\beta_{f} R_{f}^{5}\right)\right. \\
& \left.+\rho_{f}\left(\beta_{f} R_{f}^{5}-\beta_{c} R_{c}^{5}\right)+\rho_{c} \beta_{c} R_{c}^{5}\right]
\end{aligned}
$$

the subscript "in" is used instead of " $\mathrm{m}$ " to indicate that the whole interior beneath the ocean is meant. The expressions for the forced librations amplitudes and for the eigenvalues are simpler than for the generic satellite and we have

$$
\begin{aligned}
g_{s} & =\frac{4 e\left(2\left(K_{s}+K_{i n}\right) K_{i n t}+2 K_{i n} K_{s}-C_{i n} K_{s} n^{2}\right)}{C_{i n} C_{s}\left(n^{2}-\sigma_{1}^{2}\right)\left(n^{2}-\sigma_{2}^{2}\right)} \\
g_{i n} & =\frac{4 e\left(2\left(K_{s}+K_{i n}\right) K_{i n t}+2 K_{i n} K_{s}-C_{s} K_{i n} n^{2}\right)}{C_{i n} C_{s}\left(n^{2}-\sigma_{1}^{2}\right)\left(n^{2}-\sigma_{2}^{2}\right)}
\end{aligned}
$$

with

$$
\begin{aligned}
& \sigma_{1,2}^{2}= \frac{K_{i n t}\left(C_{i n}+C_{s}\right)}{C_{i n} C_{s}}+\frac{\left(C_{s} K_{i n}+C_{i n} K_{s}\right)}{C_{i n} C_{s}} \\
& \pm \frac{1}{C_{i n} C_{s}}\left[\left(C_{s}\left(K_{i n}+K_{i n t}\right)+C_{i n}\left(K_{i n t}+K_{s}\right)\right)^{2}\right. \\
&\left.\quad-4 C_{i n} C_{s}\left(K_{i n t} K_{s}+K_{i n}\left(K_{i n t}+K_{s}\right)\right)\right]^{1 / 2}
\end{aligned}
$$

The difference in shell libration amplitude $g_{s}$ computed with two liquid layers and with only one is always below $10^{-3} \%$. This proves that the liquid core has no noticeable influence on the libration of the shell, a fact that can be attribuated to the small mass of the core. We note that we also obtain similar results with an entirely liquid core.

Solution (74) for $g_{s}$ can be approximated by an appropriate order of magnitude analysis. Since the forced libration frequency is much larger than the eigenfre- 
quencies $\sigma_{1}$ and $\sigma_{2}$, we have $\sigma_{1,2}^{2} \ll n^{2}$. For all internal structure models, we then have $2\left(K_{s}+K_{i n}\right) K_{i n t}+2 K_{i n} K_{s} \ll C_{i n} K_{s} n^{2}$. Therefore,

$$
g_{s} \approx-\frac{4 e K_{s}}{n^{2} C_{s}}=-6 e \frac{\left(B_{s}-A_{s}\right)+\left(B_{o, t}-A_{o, t}\right)}{C_{s}} .
$$

Correct up to the first order in the small thickness $h_{s}$, the general expression for the shell moment of inertia

$$
C_{s}=\frac{8 \pi}{15} \rho_{s}\left[R^{5}\left(1+\frac{2}{3} \alpha_{s}\right)-R_{o}^{5}\left(1+\frac{2}{3} \alpha_{o}\right)\right]
$$

can be expressed as

$$
C_{s} \approx \frac{8 \pi}{3} \rho_{s} R^{4} h_{s}
$$

where we used that $\alpha_{s} \approx \alpha_{o}$ since $h_{s}$ is small, $\alpha_{s}$ ans $\alpha_{o}$ are the polar flattenings of the shell and of the ocean, respectively. The polar flattening $\alpha$ of any layer is defined as the relative difference $((a+b) / 2-c) /((a+b) / 2)$ of the mean equatorial and polar radii. The moment of inertia difference in the numerator in Eq. (77)

$$
\left(B_{s}-A_{s}\right)+\left(B_{t, o}-A_{t, o}\right)=\frac{8 \pi}{15}\left(\rho_{s} \beta_{s} R^{5}+\left(\rho_{o}-\rho_{s}\right) \beta_{o} R_{o}^{5}\right)
$$

in which the first term dominates the second, depends less on the ice shell thickness than does the denominator, and is independent of it for $\rho_{o}=\rho_{s}$. We then have

$$
g_{s} \approx-\frac{6}{5} e \frac{\left(\rho_{s} \beta_{s} R^{5}+\left(\rho_{o}-\rho_{s}\right) \beta_{o} R_{o}^{5}\right)}{\rho_{s} R^{4} h_{s}} .
$$

This approximate solution has the same behaviour in terms of the interior parameters as the exact solution and accounts for the dependence of $g_{s}$ on $h_{s}$ and on $\left(\rho_{o}-\rho_{s}\right)$. Nevertheless this approximation underestimates $g_{s}$ by $5-35$ 
$\mathrm{m}$. We note that $\beta_{s}=6 C_{22}+3 q / 2$ with $q=n^{2} R^{3} / G M_{\text {sat }}$. Therefore, $\beta_{s}$ is the same for all plausible internal structure models and does not depend on $h_{s}$. If $\rho_{s}=\rho_{o}$, the libration amplitude can be approximated by

$$
g_{s} \approx-\frac{6 e R \beta_{s}}{5 h_{s}} .
$$

Here, $h_{s}$ is the only parameter varying with the internal structure.

\subsection{Europa}

The mean moment of inertia and the density of Europa are compatible with a differentiated structure which consists of a metallic core, a rock mantle and an overlying water/ice layer. A mixed interior made of silicates and iron beneath a water/ice layer is also consistent with the mean moment of inertia and the density, but is not likely to exist, because of the radiogenic heating that raises the internal temperature and favors differentiation (Anderson et al. 1998). The state of the core is unknown. The absence of a permanent magnetic field (Schilling et al.2003) is compatible with both a fully liquid core and an entirely solid core. Since we have shown for Ganymede that the state of the core does not significantly influence the shell libration, we here consider that the core of Europa is solid to simplify the equations

The detection of an induced magnetic field is a strong evidence for the presence of a liquid water ocean between the mantle and the outer ice shell (Khurana et al. 1998). The reference model for Europa is then similar to the one of Ganymede with an entirely solid core but without an ice mantle (the ocean of Europa is in direct contact with the rock mantle). As for Ganymede, the core density $\rho_{c}$, the mantle density $\rho_{m}$ and the shell and the water ocean 
densities are within the ranges $5150-8000,3000-3800$ and $800-1200$ $\mathrm{kg} \mathrm{m}^{-3}$, respectively. As in Van Hoolst et al. (2008), we here take the shell thickness to be at least $5 \mathrm{~km}$, which seems to be needed to support the few kilometers topography observed on Europa (see Schenk and McKinnon, 2001), and to be at most $100 \mathrm{~km}$, well above the maximum estimated thicknesses thought to be of the order of tens of kilometers (Billings and Kattenhorn, 2005). Among our models, the water-ice layer has a maximal thickness of $\simeq 170$ $\mathrm{km}$ as in agreement with Anderson et al. (1998). For a wide and representative range of plausible internal stucture models (see Table B.3 for the values of the internal parameters), we can use the solutions (74) and (75) with $\left(B_{\text {in }}-A_{\text {in }}\right)=$ $8 \pi\left[\rho_{r}\left(\beta_{r} R_{r}^{5}-\beta_{c} R_{c}^{5}\right)+\rho_{c} \beta_{c} R_{c}^{5}\right] / 15$.

The forced libration amplitude of the shell $R g_{s}$ for our models is within the range $500-40000 \mathrm{~m}$ (Figs. 6 and 7 ) and is larger than the $\simeq 134 \mathrm{~m}$ libration of an entirely solid Europa. As for Ganymede, the libration amplitude $g_{s}$ depends mainly on the shell thickness $h_{s}$ and on the density difference $\left(\rho_{o}-\rho_{s}\right)$ and only very weakly on other interior structure parameters. The shell libration amplitude $g_{s}$ increases strongly with decreasing shell thickness (Fig. 6) due to a resonance with one of the eigenfrequencies $\sigma_{1}$ and $\sigma_{2}$, which approaches $n$ for thin ice shells as the associated normal mode represents essentialy a free libration of the ice shell (Van Hoolst et al. 2008).

The libration amplitude of the interior $R_{i} g_{i}$ is within the range $25-95 \mathrm{~m}$. The individual effect of $g_{i}$ on the time-dependent component in the degree 2 gravity coefficient is below $0.05 \%$ of the individual effect of $g_{s}$, which is of the order of $10^{-9}$ at best. 


\subsection{Callisto}

Callisto is quite similar to Ganymede in terms of size and density but its mean moment of inertia is higher. Therefore Callisto has a less differentiated structure than Ganymede. Nevertheless, the presence of an induced magnetic field implies the presence of a salty water ocean near the surface (Khurana et al. 1998), below an ice layer. With the estimated mean moment of inertia, the interior could be made of an undiferentiated mixture of ice, rocks and metal. A partially differentiated interior with a rock-metal core and an ice-rock mantle is also consistent with the moment of inertia, but such a partial differentiation is thought to lead to further differentiation because of the heat released during the differentiation process (Anderson et al. 2001a).

Although the rock and metal fractions are expected to increase with depth, the reference model for Callisto chosen here is a simple model with three layers: an ice shell overlying a water ocean and a homogenous ice-rock-metal interior. The ice shell thickness has been estimated by Zimmer et al. (2000) to be of the order of $300 \mathrm{~km}$. Here, as for Ganymede, we consider a wide range for the shell thickness from $25 \mathrm{~km}$ up to $400 \mathrm{~km}$. A shell thickness of $400 \mathrm{~km}$ is close to the maximum thickness in agreement with the moment of inertia and the density. Equations (68) and (69) are the governing libration equations. We have $\left(B_{i n}-A_{i n}\right)=8 \pi \rho_{i n} \beta_{i n} R_{i n}^{5} / 15$ since the interior is homogenous with density $\rho_{i n}$. Among a wide and representative range of models (see Table B.4 for the values of the internal parameters), the water-ice layer has a maximal thickness of $\simeq 450 \mathrm{~km}$, and the interior density is $\simeq 2300 \mathrm{~kg} \mathrm{~m}^{-3}$ (consistent with Anderson et al. 2001a). 
The forced libration amplitude of the shell at the equator, $R g_{s}$, is within the range $20-370 \mathrm{~m}$ (See Fig. 8) and is larger than the $\simeq 12 \mathrm{~m}$ libration of an entirely solid Callisto. As for Ganymede and Europa, $g_{s}$ depends mainly on $h_{s}$ and on the difference $\rho_{o}-\rho_{s}$ and very weakly on other parameters. Due to its higher eccentrity, Callisto has a shell libration amplitude very close to the one of Ganymede, despite its lower equatorial flattening.

The libration amplitude of the interior $R_{i n} g_{i n}$ is within the range $4.5-7 \mathrm{~m}$. The effect of $g_{i n}$ on the time-dependent component in the second-degree gravity coefficient is below $3 \%$ of the effect of $g_{s}$ which is of the order of $10^{-13}$ at best.

\section{$4.5 \quad I 0$}

Io most likely has a fully liquid core because of its high mantle temperature (Spohn 1997). At the core mantle boundary, the mantle temperature is higher than the melting temperature of pure iron. Light elements in the core reduce the core melting temperature with respect to a pure iron core, strengthening the argument for a liquid core. Although the core-mantle boundary temperature is model dependent, the temperature offset is large enough to ensure that the core is entirely liquid (Schubert et al. 2004). The core of Io is relatively small with respect to the cores of the terrestrial planets. By assuming a basic two-layer model consisting of a homogeneous mantle and a homogeneous core, the core size can be estimated from the observed mass, radius and moment of inertia if the composition is assumed to be known. The smallest core corresponds to a dense pure iron core and the largest core has a eutectic iron-sulfur composition. The corresponding core sizes are $657.5 \mathrm{~km}$ and $944 \mathrm{~km}$ (see also Schubert et al. 2004). 
Below the lithosphere, Io's mantle is expected to be partially melted, but an entirely liquid layer below the lithosphere is unlikely otherwise the topography could not be sustained (Webb and Stevenson 1987). Typically a partial melt of $40 \%$ is considered at which the properties of the solid phase of the mantle still dominate the properties of the liquid phase (see Schubert et al. 2004 and Stevenson 2002). Therefore, we consider that Io's mantle behaves as a solid to the rotational forcing of Jupiter.

We here use a two layer model for Io with a solid silicate mantle above a liquid core consisting predominantly of iron. The corresponding libration equation can be derived from Eqs. (62)-(64) for the generic satellite by setting $K_{c}, K_{m}, K_{m c}, K_{s c}, K_{s m}, C_{m}$ and $C_{c}$ equal to zero. The constant $K_{s}$ is the same as for the entire body, so that we write it as $K$ :

$$
K=\frac{3}{2} n^{2}\left[\left(B_{m}-A_{m}\right)+\left(B_{f, t}-A_{f, t}\right)\right]=\frac{3}{2} n^{2}(B-A),
$$

where the subscripts $s, o$ have been replaced by $m, f$. The libration equation for the mantle is then given by:

$$
C_{m} \ddot{\gamma}_{m}+2 K \gamma_{m}=4 e K \sin M
$$

The forced libration amplitude and free libration frequency can be expressed

as

$$
\begin{aligned}
g_{m} & =-\frac{4 e K}{C_{m}\left(n^{2}-\sigma^{2}\right)}, \\
\sigma^{2} & =2 K / C_{m} .
\end{aligned}
$$

For an iron density of $8000 \mathrm{~kg} \mathrm{~m}^{-3}$, the libration amplitude $R g_{m}$ is $272.5 \mathrm{~m}$ and for a eutectic iron-sulfur core with a density of $5150 \mathrm{~kg} \mathrm{~m}^{-3}, R g_{m}=285.2$ m. The amplitude increase with respect to a solid Io is only a few percent 
because the core is small with respect to the mantle.

Since $B-A \ll C_{m}$, the free libration frequency $\sigma \ll n$ and the libration amplitude can approximately be expressed as

$$
g_{m} \approx-6 e \frac{B-A}{C_{m}}
$$

For Io, the approximate solution (81) cannot be used since $\rho_{m} \neq \rho_{c}$ and since the mantle is not a thin layer (but solution (77) is valid though). Although $\alpha_{m}$ and $\alpha_{f}$ are quite different because of the large thickness of the mantle, the polar moment of inertia of the mantle $C_{m}$

$$
C_{m}=\frac{8 \pi}{15} \rho_{m}\left[R^{5}\left(1+\frac{2}{3} \alpha_{m}\right)-R_{f}^{5}\left(1+\frac{2}{3} \alpha_{f}\right)\right]
$$

can be expressed as

$$
C_{m} \approx \frac{8 \pi}{15} \rho_{m}\left[R^{5}-R_{f}^{5}\right]
$$

since $\alpha_{m}$ and $\alpha_{f}$ are $\ll 1$. Therefore we rewrite solution (87) as

$$
g_{m} \approx \frac{-45 e M R^{2} C_{22}}{\pi \rho_{m}\left(R^{5}-\left(R-h_{m}\right)^{5}\right)}
$$

By expressing $\rho_{m}$ in terms of $h_{m}, M$ and $I$, we have

$$
g_{m} \approx \frac{120 C_{22} \text { e } M\left(h_{m}-2 R\right) R^{5}}{\left(5 I-2 M\left(h_{m}-R\right)^{2}\right)\left(h_{m}^{4}-5 h_{m}^{3} R+10 h_{m}^{2} R^{2}-10 h_{m} R^{3}+5 R^{4}\right)}
$$

In this expression, $h_{m}$ is the only internal parameter varying with the internal structure model. Even if this approximate solution underestimates the libration amplitude of the mantle, it accounts for the dependence of $g_{m}$ on the mantle thickness $h_{m}$, through $C_{m}$. 


\section{Discussion and conclusion}

We have developed a theoretical model to describe the influence of up to two global internal liquid layers on the libration of the Galilean satellites or any other satellite in 1:1 spin-orbit resonance. In our model, the libration amplitude of each layer of a satellite is the result of the gravitational torque of Jupiter on the layer and of the interaction with the other layers through gravitational and pressure torques. We have derived analytical solutions for the libration amplitude of each solid layer and shown that the liquid layers do not take part in the libration.

For Io, the presence of the liquid core increases the libration of the mantle by only a few percent with respect to an entirely solid Io because the core is relatively small with respect to the mantle. On the other hand, the presence of a water ocean close to the surface of Europa, Ganymede, and Callisto increases strongly the amplitude of the forced libration of the ice shell, but the presence of a second global internal liquid layer in the form of a liquid iron core, for Europa and Ganymede, has no noticeable effect on the shell libration amplitude. We have shown that the shell libration depends essentially on the shell thickness and to a minor extent on the density difference between the ocean and the ice shell (See Eq. 81). Therefore libration observations cannot only be used to prove the existence of a subsurface ocean but also to constrain the shell thickness and density difference between the ocean and the shell.

For Europa, Ganymede, and Callisto, the error on the thickness of the ice shell can be estimated from the observational error on the shell libration amplitude. When we assume that the ocean and the shell have equal density $\left(\rho_{o}=\rho_{s}\right)$, 
this can most simply be done by using Eq. (82). The error on the surface layer thickness $\Delta h_{s}$ from an observational error $\Delta g_{s}$ on $g_{s}$ is given by

$$
\Delta h_{s} \approx\left|\frac{\Delta g_{s}}{\partial_{h_{s}} g_{s}}\right|=\left|\frac{h_{s}}{g_{s}} \Delta g_{s}\right| .
$$

A precision $\Delta g_{s}$ of several meters is expected on the librations measurements for Europa and Ganymede with the NASA/ESA EJSM mission planned to deliver an orbiter both around Europa and Ganymede. The error on the ice shell thickness determination is represented in Fig. 9 for an error of $1 \mathrm{~m}$ on the measurement of the shell libration at the equator. The error increases with the ice shell thickness. The errors for Ganymede and Callisto are similar since their libration amplitudes are also similar. For Europa, $\Delta h_{s}$ is about 10 times smaller than for Ganymede and Callisto at a given shell thickness, since its libration amplitude $g_{s}$ is about 10 times higher. If the shell and ocean density were to be equal, the shell thickness could be estimated with a precision of about $100 \mathrm{~m}$ for Europa and a few km for Ganymede and Callisto. Although these results seem very encouraging, the dependence of the libration amplitude of the shell $g_{s}$ on the density difference $\rho_{o}-\rho_{s}$ introduces a much larger uncertainty. For example, at shell thicknesses around $50 \mathrm{~km}$, an uncertainty on the density difference $\rho_{o}-\rho_{s}$ of $200 \mathrm{~kg} \mathrm{~m}^{-3}$ will induce an additional error on the shell thickness $h_{s}$ of about $10 \mathrm{~km}$ for europa (Fig. 7). At $h_{s} \approx 100 \mathrm{~km}$ for Ganymede and Callisto, the addtional error is about $20 \mathrm{~km}$ (Figs. 5 and 8).

For Io, the error on the thickness of the mantle $\Delta h_{m}$ can be estimated from the observational error $\Delta g_{m}$ on the mantle libration amplitude $g_{m}$ by using Eq. (91). As can be seen from Fig. 9, the error on the mantle thickness determination is several tens of kilometers. 
Since only the amplitude of the surface layer libration depends on the thickness of the surface layer, the libration of the surface layer is more relevant to measure than the libration of the other solid layers. Furthermore, it is the only libration observable by imaging. Librations can also be measured through their influence on the second-degree gravity field coefficient $C_{22}$. We have shown that the effect of the surface layer on $C_{22}$ is at least an order of magnitude larger than the effect of the other solid layers. Therefore the measurements of the time-variable component of $C_{22}$ can be interpreted in terms of the surface layer libration only.

In this study, we have made several approximations. First, the different layers are assumed to be homogeneous in density. This assumption is most likely satisfied since the layers are approximately homogeneous in composition and the effect of pressure on density is small for the relatively small satellites (Sohl et al. 2002). Moreover, the inclusion of the pressure effect and composition effects like the dependence of the fraction of rocks and metal on depth in the interior modeling would almost not change our main results since the shell and ocean density are not strongly affected by them. A more critical assumption is that of hydrostatic equilibrium, which we have used to calculate the equatorial flattening of the layers. The validity of this hypothesis is difficult to judge for satellites, although indications in favor of hydrostatic equilibrium exist such as the ratio $J_{2} / C_{22}$, which is consistent with the hydrostatic value of $10 / 3$ for Io, and the fact that the shape of many but not all large and medium-sized satellites seems to be consistent to first order with that of a relaxed satellite in hydrostatic equilibrium (e.g. Thomas et al. 2007, Van Hoolst et al. 2008, 2009). Moreover, our result that the amplitude of the shell libration depends on the shell thickness and on the density difference between the ocean and the 
shell (see Eq. 81) is valid also for non-hydrostatic equatorial flattenings as long as they are not larger than about 5, 15 and 25 times their hydrostatic values for Europa, Ganymede and Callisto, respectively. The shape of the ice shell of the Galilean satellites may deviate substantially from the hydrostatic form due to lateral variations in tidal dissipation and surface temperature, which can induce variations of the shell thickness of about $5 \mathrm{~km}$ for Europa (Tobie et al. 2003). Since maxima in shell thickness are obtained at the sub-Jovian and anti-Jovian points and the minima in the perpendicular direction, these effects increase the shell equatorial flattening and so also the shell libration amplitude (see Eq. 82). Other effects could decrease the libration amplitude; in particular deformation of the layers due to the librations themselves, the tidal potential and the misalignement of the layers, which we have neglected here on the basis of order of magnitude analyses, absorbs part of the angular momentum. These, and other, effects require further study.

\section{Acknowledgments}

R.-M. Baland is a Research Fellow of the Fonds pour la formation à la Recherche dans l'Industrie et dans l'Agriculture (FRIA), Belgium. We acknowledge S. J. Peale for his useful comments which helped us to improve the paper. 


\section{A Gravitational torques}

A.1 Internal gravitational torque exerted on an internal satellite layer by another layer further away from the center of mass of the satellite

In our generic satellite each layer is acted on by a torque exerted by the satellite masses outside that layer. For homogeneous or stratified layers, the coupling is different from zero only when the layers are not aligned. Here we derive an expression for the torque exerted on a satellite layer by another satellite layer located further away from the center of mass of the satellite. As an example, the mantle ('inner layer') undergoes a torque caused by the shell and the top part of the ocean (together constituting the 'outer layer'). The z-component of such a torque is given by:

$$
\Gamma_{g, I}=-\int_{V_{I}}\left[\vec{r} \wedge \rho_{I} \nabla \Phi_{O}(r, \theta, \varphi)\right]_{z} d V
$$

where $V_{I}$ and $\rho_{I}$ are the volume and the density of the 'inner layer' and $\Phi_{O}$ the gravitational potential of the 'outer layer'. Since only those parts of the gravitational potential that depend on the longitude lead to a torque, we neglect the spherically symmetric and zonal contributions and only consider

$$
\Phi_{O}(r, \theta, \varphi)=-\frac{2 \pi G}{15} r^{2}\left[\int_{O} \rho(r) \frac{\partial \beta(r)}{\partial r} d r\right] R_{22}(\theta, \varphi)
$$

where

$$
R_{22}(\theta, \varphi)=3 \sin ^{2} \theta \cos 2 \varphi
$$

is the real sectorial spherical harmonic of degree 2. Radius $r$, colatitude $\theta$ and longitude $\varphi$ are the spherical coordinates of a point of the 'inner layer' defined 
in the principal frame of the 'outer layer'. By using a consequence of Gauss' theorem

$$
\int_{V} \vec{r} \wedge \nabla f d V=\int_{S} f \vec{r} \wedge \hat{n} d S
$$

and by assuming that the density in the 'inner layer' is constant, we have

$$
\begin{aligned}
\Gamma_{g, I}= & -\int_{S_{I t}}\left[\vec{r}_{I t} \wedge \vec{n}_{I t}\right]_{z} \rho_{I} \Phi_{O}\left(r_{I t}, \theta, \varphi\right) d S \\
& +\int_{S_{I b}}\left[\vec{r}_{I b} \wedge \vec{n}_{I b}\right]_{z} \rho_{I} \Phi_{O}\left(r_{I b}, \theta, \varphi\right) d S
\end{aligned}
$$

with $S_{I t}$ and $S_{I b}$ the top and the bottom surfaces of the 'inner layer' (triaxial ellipsoids with mean radius $R_{I t}$ and $R_{I b}$ ), and $\vec{n}_{I t}$ and $\vec{n}_{I b}$ the outward unit normals on $S_{I t}$ and $S_{I b}$,

$$
\begin{aligned}
\vec{n}_{I t} & =\frac{\partial_{\theta} \vec{r}_{I t} \wedge \partial_{\varphi} \vec{r}_{I t}}{\left\|\partial_{\theta} \vec{r}_{I t} \wedge \partial_{\varphi} \vec{r}_{I t}\right\|}, \\
\vec{n}_{I b} & =\frac{\partial_{\theta} \vec{r}_{I b} \wedge \partial_{\varphi} \vec{r}_{I b}}{\left\|\partial_{\theta} \vec{r}_{I b} \wedge \partial_{\varphi} \vec{r}_{I b}\right\|}
\end{aligned}
$$

Here, $\vec{r}_{I t}$ and $\vec{r}_{I b}$ are the position vectors of the points located on $S_{I t}$ and $S_{I b}$, which can be expressed in cartesian coordinates as

$$
\begin{aligned}
& \vec{r}_{I t}=\left(r_{I t} \sin \theta \cos \varphi, r_{I t} \sin \theta \sin \varphi, r_{I t} \cos \theta\right), \\
& \vec{r}_{I b}=\left(r_{I b} \sin \theta \cos \varphi, r_{I b} \sin \theta \sin \varphi, r_{I b} \cos \theta\right),
\end{aligned}
$$

and $r_{I t}$ and $r_{I b}$ are the distance to the satellite center of a point located on the triaxial ellipsoid $S_{I t}$ and $S_{I b}$, respectively.

If the inner layer is aligned with the outer layer, we have

$$
\begin{aligned}
& r_{I t}=R_{I t}\left(1-\frac{2}{3} \alpha_{I t} R_{20}+\frac{1}{6} \beta_{I t} R_{22}\right), \\
& r_{I b}=R_{I b}\left(1-\frac{2}{3} \alpha_{I b} R_{20}+\frac{1}{6} \beta_{I b} R_{22}\right),
\end{aligned}
$$


where

$$
R_{20}=\frac{1}{2}\left(3 \cos ^{2} \theta-1\right)
$$

and $\alpha$ and $\beta$ are the polar and equatorial flattenings, defined as the relative differences $(a-b) / a$ and $((a+b) / 2-c) /((a+b) / 2)$, respectively, with $a>b>c$, the radii in the direction of the principal axis of the layer. However, if the inner layer is shifted by an angle $\zeta$ in longitude with respect to the outer layer, the radial distances can be expressed as

$$
\begin{aligned}
r_{I t} & =R_{I t}\left(1-\frac{2}{3} \alpha_{I t} R_{20}+\frac{\beta_{I t}}{6} \cos 2 \zeta R_{22}+\frac{\beta_{I t}}{6} \sin 2 \zeta S_{22}\right), \\
r_{I b} & =R_{I b}\left(1-\frac{2}{3} \alpha_{I b} R_{20}+\frac{\beta_{I b}}{6} \cos 2 \zeta R_{22}+\frac{\beta_{I b}}{6} \sin 2 \zeta S_{22}\right), \\
S_{22} & =3 \sin ^{2} \theta \sin 2 \varphi .
\end{aligned}
$$

The vectorial products in Eq. (A.5) can then, by keeping only the terms depending on the longitude (other terms do not lead to a torque) be written correct up to the first order in $\beta$ as

$$
\begin{aligned}
& {\left[\vec{r}_{I t} \wedge \vec{n}_{I t}\right]_{z}=-R_{I t} \beta_{I t} \sin ^{2} \theta \sin 2(\zeta-\varphi),} \\
& {\left[\vec{r}_{I b} \wedge \vec{n}_{I b}\right]_{z}=-R_{I b} \beta_{I b} \sin ^{2} \theta \sin 2(\zeta-\varphi) .}
\end{aligned}
$$

Since $\zeta=\phi_{I}-\phi_{O}$, the difference between the rotation angles of the two layers, the torque (A.1) can be expressed as

$$
\Gamma_{g, I}=\frac{32 \pi^{2} G}{75}\left[\int_{I} \rho_{I} \frac{\partial\left[r^{5} \beta(r)\right]}{\partial r} d r\right]\left[\int_{O} \rho(r) \frac{\partial \beta(r)}{\partial r} d r\right] \sin 2\left(\phi_{O}-\phi_{I}\right) .
$$

The torque depends on twice the difference in orientation $\phi_{O}-\phi_{I}$ since it is maximum when the axes make an angle of $45^{\circ}$. The dependence on the flattening, density and size of the layers is factorized into a factor depending on the 'inner layer' and a factor linked to the 'outer layer'. The torque can 
also be written in terms of $\left(B_{I}-A_{I}\right)$, the difference of equatorial moments of inertia of the internal layer, as

$$
\Gamma_{g, I}=\frac{4 \pi G}{5}\left(B_{I}-A_{I}\right)\left[\int_{O} \rho(r) \frac{\partial \beta(r)}{\partial r} d r\right] \sin 2\left(\phi_{O}-\phi_{I}\right)
$$

This Eq. (A.19) is more general than Eq. (A.18) since it is also valid for a non-homogenous internal layer, as has been shown by e.g. Dumberry (2008) in the particular case of the gravitational torque on the inner core of the Earth due to the outer core, mantle and crust.

A.2 Internal gravitational torque exerted on a satellite layer by another layer closer to the center of mass

Each satellite layer, except for the innermost layer, is acted on by a gravitational torque exerted by the masses located closer to the center than the layer considered. For example in our generic satellite, the solid core and the bottom part of the fluid core ('inner layer') exert a torque on the shell ('outer layer'). The z-component of such a torque is given by

$$
\Gamma_{g, O}=-\int_{V_{O}}\left[\vec{r} \wedge \rho_{O} \nabla \Phi_{I}(r, \theta, \varphi)\right]_{z} d V
$$

with $V_{O}$ and $\rho_{O}$ the volume and the density of the 'outer layer' and $\Phi_{I}$ the gravitational potential of the 'inner layer' depending on the longitude (other terms do not lead to a torque). An expression for the torque $\Gamma_{g, O}$ can be derived in the same way as for $\Gamma_{g, I}$ above, but since the torque of an 'inner layer' on an 'outer layer' is equal and opposite to the torque of an 'outer layer' on an 'inner layer', $\Gamma_{g, O}=-\Gamma_{g, I}$, we immediately have 


$$
\Gamma_{g, O}=-\frac{4 \pi G}{5}\left(B_{I}-A_{I}\right)\left[\int_{O} \rho(r) \frac{\partial \beta(r)}{\partial r} d r\right] \sin 2\left(\phi_{O}-\phi_{I}\right) .
$$

\section{B Pressure torques}

In Sect. 3.3, we have decomposed the pressure in a contribution due to the internal potential and a contribution due to the external potential (see Eq. (36)). Therefore, the pressure torque could be decomposed in the same way. Here, we first consider the pressure torque related to the internal gravitational potential and next the pressure torque related to the Jupiter potential.

B.1 Pressure torque related to the internal gravitational potential on an internal layer and caused by another layer further away from the center of mass of the satellite

The part of the pressure torque on an internal satellite layer ('inner layer'), due to a satellite layer further away from the center ('outer layer'), related to the internal gravitational potential is given by

$$
\begin{aligned}
\Gamma_{p g, I}= & -\int_{S_{I t}}\left[\vec{r}_{I t} \wedge \vec{n}_{I t}\right]_{z} P_{g}\left(r_{I t}, \theta, \varphi\right) d S \\
& +\int_{S_{I b}}\left[\vec{r}_{I b} \wedge \vec{n}_{I b}\right]_{z} P_{g}\left(r_{I b}, \theta, \varphi\right) d S .
\end{aligned}
$$

By using applying Gauss' theorem, the condition of hydrostatic equilibrium

$$
\begin{aligned}
& \nabla P_{g}\left(r_{I t}, \theta, \varphi\right)=-\rho_{I t} \nabla \Phi_{O}\left(r_{I t}, \theta, \varphi\right), \\
& \nabla P_{g}\left(r_{I b}, \theta, \varphi\right)=-\rho_{I b} \nabla \Phi_{O}\left(r_{I b}, \theta, \varphi\right),
\end{aligned}
$$

where $\rho_{I t}$ and $\rho_{I b}$ are the density of the fluid located at the top and bottom surfaces of the 'inner layer', and again applying Gauss' theorem, we have: 


$$
\begin{aligned}
\Gamma_{p g, I}= & \int_{S_{I t}}\left[\vec{r}_{I t} \wedge \vec{n}_{I t}\right]_{z} \rho_{I t} \Phi_{O}\left(r_{I t}, \theta, \varphi\right) d S \\
& -\int_{S_{I b}}\left[\vec{r}_{I b} \wedge \vec{n}_{I b}\right]_{z} \rho_{I b} \Phi_{O}\left(r_{I b}, \theta, \varphi\right) d S .
\end{aligned}
$$

By using a similar calculation as is the Sect. A.1, we have:

$$
\begin{aligned}
\Gamma_{p g, I}= & \frac{32 \pi^{2} G}{75}\left[\rho_{I b} R_{I b}^{5} \beta_{I b}-\rho_{I t} R_{I t}^{5} \beta_{I t}\right]\left[\int_{O} \rho(r) \frac{\partial \beta(r)}{\partial r} d r\right] \\
& \times \sin 2\left(\phi_{O}-\phi_{I}\right) .
\end{aligned}
$$

Since the core has no bottom surface, the torque on the core is obtained by taking the first term in Eq. (B.1) or, equivalently, by setting $\rho_{c l b}$ equal to zero in Eq. (B.6).

B.2 Pressure torque related to the internal gravitational potential on an internal layer and caused by another layer closer to the center of mass of the satellite

Since the surface torque applied on the masses internal to a surface due to the masses external to this surface is equal and opposite to the torque exerted by the masses internal to the surface on the masses external to the surface, it may seem that an expression for the pressure torque on an 'outer layer' caused by an 'inner layer' can simply be obtained by using the principle of action and reaction as for the internal gravitational torque. However, the pressure torque related to an 'inner layer' on an 'outer layer' is not equal and opposite in general to that on the 'inner layer' because the surfaces involved are not the same. Therefore, we do not use the principle of action and reaction and derive an expression for the pressure torque due to the internal gravitational potential on an 'outer layer' caused by an 'inner layer' as in the previous section. We 
have

$$
\begin{aligned}
\Gamma_{p g, O}= & -\int_{S_{O t}}\left[\vec{r}_{O t} \wedge \vec{n}_{O t}\right]_{z} P_{g}\left(r_{O t}, \theta, \varphi\right) d S \\
& +\int_{S_{O b}}\left[\vec{r}_{O b} \wedge \vec{n}_{O b}\right]_{z} P_{g}\left(r_{O b}, \theta, \varphi\right) d S
\end{aligned}
$$

with $S_{O t}$ and $S_{O b}$ the top and the bottom surfaces of the 'outer layer' (triaxial ellipsoids with mean radius $R_{O t}$ and $\left.R_{O b}\right), \vec{n}_{O t}$ and $\vec{n}_{O b}$ the outward unit normals on $S_{O t}$ and $S_{O b} \cdot \vec{r}_{O t}$ and $\vec{r}_{O b}$ are the position vector of the points located on $S_{O t}$ and $S_{O b}$. Since

$$
\begin{aligned}
& \nabla P_{g}\left(r_{O t}, \theta, \varphi\right)=-\rho_{O t} \nabla \Phi_{I}\left(r_{O t}, \theta, \varphi\right), \\
& \nabla P_{g}\left(r_{O b}, \theta, \varphi\right)=-\rho_{O b} \nabla \Phi_{I}\left(r_{O b}, \theta, \varphi\right),
\end{aligned}
$$

where $\rho_{O t}$ and $\rho_{O b}$ are the density of the fluid located at the top and bottom surfaces of the 'outer layer', $\Phi_{I}$ is the gravitational potential of the 'inner layer' depending on the longitude (other terms do not lead to a torque)

$$
\Phi_{I}(r, \theta, \varphi)=-\frac{2 \pi G}{5 r^{3}}\left[\int_{I} \rho(r) \frac{\partial\left[r^{5} \beta(r)\right]}{\partial r} d r\right] \sin ^{2} \theta \cos (2 \varphi-2 \zeta)
$$

Radius $r$, colatitude $\theta$ and longitude $\varphi$ are the spherical coordinates of a point of the 'outer layer' defined in the principal frame of this 'outer layer'. $\zeta=\phi_{I}-\phi_{O}$ is the longitude shift of the 'inner layer' with respect to the 'outer layer'. By application of Gauss' theorem, we then have:

$$
\begin{aligned}
\Gamma_{p g, O}= & \rho_{O t} \int_{S_{O t}}\left[\vec{r}_{O t} \wedge \vec{n}_{O t}\right]_{z} \Phi_{I}\left(r_{O t}, \theta, \varphi\right) d S \\
& -\rho_{O b} \int_{S_{O b}}\left[\vec{r}_{O b} \wedge \vec{n}_{O b}\right]_{z} \Phi_{I}\left(r_{O b}, \theta, \varphi\right) d S .
\end{aligned}
$$

By using a similar calculation as before, we finally have

$$
\Gamma_{p g, O}=-\frac{4 \pi G}{5}\left(B_{I}-A_{I}\right)\left(\beta_{O b} \rho_{O b}-\beta_{O t} \rho_{O t}\right) \sin 2\left(\phi_{O}-\phi_{I}\right) .
$$


There is no fluid layer above the shell, because we neglect the presence of the weak atmosphere of the Galilean satellites. Therefore, the torque on the shell is obtained by taking the second term in Eq. (B.7) or, equivalently, by setting $\rho_{O t}$ equal to zero in Eq. (B.12).

B.3 Pressure torque due to the external gravitational potential on any internal satellite layer

The part of the pressure torque due to the external potential on any layer is given by

$$
\begin{aligned}
\Gamma_{p e, l}= & -\int_{S_{l t}}\left[\vec{r}_{l t} \wedge \vec{n}_{l t}\right]_{z} P_{e}\left(r_{l t}, \theta, \varphi\right) d S \\
& +\int_{S_{l b}}\left[\vec{r}_{l b} \wedge \vec{n}_{l b}\right]_{z} P_{e}\left(r_{l b}, \theta, \varphi\right) d S .
\end{aligned}
$$

with $S_{l t}$ and $S_{l b}$ the top and the bottom surfaces of the layer (triaxial ellipsoids with mean radius $R_{l t}$ and $R_{l b}$ ), $\vec{n}_{l t}$ and $\vec{n}_{l b}$ the outward unit normals on $S_{l t}$ and $S_{l b} \cdot \vec{r}_{l t}$ and $\vec{r}_{l b}$ are the position vector of the points located on $S_{l t}$ and $S_{l b}$.

Since

$$
\begin{aligned}
& \nabla P_{e}\left(r_{l t}, \theta, \varphi\right)=-\rho_{l t} \nabla W\left(r_{l t}, \theta, \varphi\right), \\
& \nabla P_{e}\left(r_{l b}, \theta, \varphi\right)=-\rho_{l b} \nabla W\left(r_{l b}, \theta, \varphi\right),
\end{aligned}
$$

where $\rho_{l t}$ and $\rho_{l b}$ are the density of the fluid located at the top and bottom surfaces of the layer, with $W$ the gravitational potential of Jupiter depending on the longitude (other terms do not lead to a torque)

$$
W(r, \theta, \varphi)=-\frac{3}{4} \frac{G M_{p}}{d^{3}} r^{2} \sin ^{2} \theta \cos 2\left(\varphi-\psi_{l}\right)
$$

$r, \theta$ and $\varphi$ are the spherical coordinates of a point of the layer defined in a the principal frame of this layer and $\psi_{l}$ is the angle between the long axis of the 
layer and the direction from the satellite to Jupiter. By application of Gauss' theorem, we have

$$
\begin{aligned}
\Gamma_{e g, l}= & \int_{S_{l t}}\left[\vec{r}_{l t} \wedge \vec{n}_{l t}\right]_{z} \rho_{l t} W\left(r_{i l t}, \theta, \varphi\right) d S \\
& -\int_{S_{l b}}\left[\vec{r}_{l b} \wedge \vec{n}_{l b}\right]_{z} \rho_{l b} W\left(r_{l b}, \theta, \varphi\right) d S
\end{aligned}
$$

and finally obtain

$$
\Gamma_{p g, e l}=\frac{4 \pi}{5}\left[R_{l b}^{5} \beta_{l b} \rho_{l b}-R_{l t}^{5} \beta_{l t} \rho_{l t}\right] \frac{G M_{p}}{d^{3}} \sin 2 \psi_{l}
$$




\section{References}

[1] Anderson, J. D., Lau, E. L, Sjogren, W. L., Schubert, Moore, W. B., 1996. Gravitational constraints on the internal structure of Ganymede. Nature, $384,541-543$

[2] Anderson, J. D., Schubert, G., Jacobson, R. A., Lau, E. L., Moore, W. B.,

Sjogren, W. L., 1998. Europa's Differentiated Internal Structure: Inferences from Four Galileo Encounters. Science, 281, 2019-+

[3] Anderson, J. D., Jacobson, R. A., McElrath, T. P, Moore, W. B., Schubert, G., Thomas, P. C, 2001a. Shape, Mean Radius, Gravity Field, and Interior Structure of Callisto. Icarus, 153, 157-161

[4] Anderson, J. D., Jacobson, R. A., Lau, E. L., Moore, W. B., Schubert, G., 2001b. Io's gravity field and interior structure. Journal of Geophysical Research, 106, 32,963-32,969

[5] Billings, S. E., Kattenhorn, S. A., 2005. The great thickness debate: Ice shell thickness models for Europa and comparisons with estimates based on flexure at ridges. Icarus, 177, 397-412

[6] Bills, B.G., 2005. Free and forced obliquities of the Galilean satellites of Jupiter. Icarus 175, 233-247.

[7] Bills, B.G., Nimmo, F., 2008. Forced obliquity and moments of inertia of Titan. Icarus 196, 293-297.

[8] Buffett, B.A., 1996. Gravitational oscillations in the length of day. Geophys. Res. Lett. 23(17), 2279-2282.

[9] Cayley, A., 1861. Tables of the developments of functions in the theory of elliptic motion. Mem. R. Astron. Soc. 29, 191-306.

[10] Chao, B. F., Liu, H. S, Dong, D. N, Herring, T. A., 1991. Libration in the earth's rotation. Geophys. Res. Lett. 18(11), 2007-2010. 
[11] Comstock, R.L., Bills, B.G., 2003. A solar system survey of forced librations in longitude. J. Geophys. Res. 108(E9), 5100, doi:10.1029/2003JE00210.

[12] Dumberry, M., 2008, Gravitational torque on the inner core and decadal polar motion, Geophys. J. Int. 172, 903-920.

[13] Fortes, A. D., Grindrod, P.M., Trickett, S.K., Vočadlo L., 2007. Ammonium sulfate on titan: Possible origin and role in cryovolcanism. Icarus, 188, 139-153, doi:10.1016/j.icarus.2006.11.002.

[14] Hansen, P.A., 1855. Abhandlungen der Mathematisch-Physischen Class der Koniglich Sachsischen Gesellschaft der Wissenschaften. Leipzig, Vol. 2, $515-523$.

[15] Hinderer, J., Legros, H., Amalvict, M., 1982. A search for Chandler and nearly diurnal wobbles using Liouville equations. Geophys. J. R. Astron. Soc. $71,303-332$.

[16] Hough, S.S., 1895, The oscillations of a rotating ellipsoidal shell containing fluid, Phil. Trans. Roy. Soc. London A 185, 469-506.

[17] Hughes, S., 1981. The computation of tables of Hansen coefficients. Cel. Mech 25, 101-107.

[18] Iess, L., Armstrong, J.W, diBenedetto, M., Graziani, A. Mackenzie, R., Racioppa, P., Rappaport, N., Tortora, P., 2007. The determination of Titan gravity field from Doppler tracking of the Cassini spacecraft. in: Proceedings of the 20th International Symposium on Space Flight Dynamics, NASA/CP-2007-214158.

[19] Jeffreys, H., 1952. The Earth. Its origin, history and physical constitution. Cambridge University Press, Cambridge, UK.

[20] Karatekin, Ö., Van Hoolst, T. , T., 2008. The effect of internal gravitational coupling on Titan's non-synchronous rotation. Geophys. Res. Lett., 
$35,16202-+$.

[21] Khurana, K. K., Kivelson, M. G., Stevenson, D. J., 1998. Induced magnetic fields as evidence for subsurface oceans in Europa and Callisto. Nature, $395,777-780$.

[22] Lainey, V., Duriez, L.;,Vienne, A., 2004a. New accurate ephemerides for the Galilean satellites of Jupiter. I. Numerical integration of elaborated equations of motion. Astron. Astrophys., 420, 1171-118.

[23] Lainey, V., Arlot, J. E., Vienne, A., 2004b. New accurate ephemerides for the Galilean satellites of Jupiter. II. Fitting the observations. Astron. Astrophys., 420, 1171-118.

[24] Lainey, V., Arlot, J. E., Vienne, A., Karatekin, Ö., Van Hoolst, T., 2009. Strong tidal dissipation in Io and Jupiter from astrometric observations. Nature, 459, 957-959.

[25] Mathews, P.M., Buffett, B.A., Herring, T.A., Shapiro, I.I., 1991. Forced nutations of the Earth: influence of inner core dynamics. 1. Theory. J. Geophys. Res. 96, 8219-8242.

[26] Moore, W. B., Schubert, G., 2000. NOTE: The Tidal Response of Europa. Icarus. 147, 317-319.

[27] Moore, W. B., Schubert, G., 2003. The tidal response of Ganymede and Callisto with and without liquid water oceans. Icarus. 166, 223-226.

[28] Moritz, H., Mueller, I.I., 1987. Earth rotation. Theory and Observation. Ungar New York, USA.

[29] Murray, C.D., Dermott, S.F., 1999. Solar Sysytem Dynamics. Cambridge University Press.

[30] Noir, J., Hemmerlin, F., Wicht, J., Baca, S.M., Aurnou, J.M., 2009, An experimental and numerical study if librationally driven flow in planetary cores and oceans, Phys. Earth Plan. Int. 173, 141-15. 
[31] Persi del Marmo, P., Iess, L., Picardi, G., Seu, R., Bertotti, B., 2007. The Determination Of Titan's Rotational State From Cassini SAR Images. Bull. Am. Astron. Soc. 39, 543.

[32] Peale, S. J., 1988. The rotational dynamics of Mercury and the state of its core. Mercury. University of Arizona Press. 461-493

[33] Peale, S. J., Phillips, R.J., Solomon, S.C., Smith, D.E., Zuber, M.T., 2002. A procedure tfor determining the nature of Mercury's core. Meteoritics Planet. Sci. 37, 1269-1283.

[34] Plummer, H.C. 1918. An introductory treatise on dynamical astronomy. Cambridge University Press, Cambridge, UK.

[35] Poincaré, H., 1910. Sur la précession des corps déformables. Bull. Astron. $27,321-356$.

[36] Rambaux, N., Van Hoolst, T., Dehant, V., Bois, E., 2006. Inertial coremantle coupling and libration of Mercury. Astron. Astrophys. 468, 711-719.

[37] Rambaux, N., Castillo-Rogez, J.C., Williams, J.G., Karatekin, Ö., 2010, The librational response of Enceladus, GRL, in press.

[38] Schenk, P. M., McKinnon, W. B., 2001. Topographic Variability on Europa from Galileo Stereo Images. Lunar. Planet. Sci. XXXII, Abstract 2078. [39] Schubert, G., Anderson, J. D., Spohn, T., McKinnon, W. B., 2004. Interior composition, structure and dynamics of the Galilean satellites. Jupiter. The Planet, Satellites and Magnetosphere, 281-306.

[40] Sohl, F., Spohn, T., Breuer, D., Nagel, K., L, 2002. Implications from Galileo Observations on the Interior Structure and Chemistry of the Galilean Satellites. Icarus. 157, 104-119.

[41] Sohl, F., Hussmann, H., Schwentker, B., Spohn, T., Lorenz, R. D., 2003. Interior structure models and tidal Love numbers of Titan. J. Geophys. Res. 108,5130 . 
[42] Stevenson, D. J., 2002. Tidal Response and Stability of Two-phase Media: Implications for Io, Europa and Titan. AGU Fall Meeting Abstracts. C10+.

[43] Stiles, B.W., Kirk, R.L., Lorenz, R.D., Hensley, S., Lee, E., Ostro, S.J., Allison, M.D., Callahan, P.S., Gim, Y., Iess, L., Persi del Marmo, P., Hamilton, G., Johnson, W.T.K., West, R.D. 2008. Determining Titan's Spin State from Cassini Radar Images. Astron. J. 135(5), 1669-1680, 10.1088/0004$6256 / 135 / 5 / 1669$.

[44] Thomas, P. C., Burns, J. A., Helfenstein, P., Squyres, S., Veverka, J., Porco, C., Turtle, E. P., McEwen, A., Denk, T., Giese, B., Roatsch, T., Johnson, T. V., Jacobson, R. A., 2007. Shapes of the saturnian icy satellites and their significance. Icarus. 190, 573-584.

[45] Tobie, G., Choblet, G., Sotin, C., 2003. Tidally heated convection: Constraints on Europa's ice shell thickness. Journal of Geophysical Research (Planets), 108, 5124-+.

[46] Van Hoolst, T., 2007. The rotation of the terrestrial planets. Treatise on Geophysics, Vol.10: Planets and Moons, pp. 123-164, doi: 10.1007/s11214$007-9202-6$

[47] Van Hoolst, T., Dehant, V. 2002. Influence of triaxiality and second-order terms in flattenings on the rotation of terrestrial planets: I. Formalism and rotational normal modes. Phys. Earth Planet. Int. 134, 17-33.

[48] Van Hoolst, T., Rambaux, N., Karatekin, Ö., Dehant, V., Rivoldini, A., 2008. The librations, shape, and icy shell of Europa. Icarus 195/1, 386-399..

[49] Van Hoolst, T., Rambaux, N., Karatekin, Ö., Baland, R.-M., 2009. The effect of gravitational and pressure torques on Titan's length-of-day variations. Icarus, 200, 256-264.

[50] Vočadlo, L., 2007. Mineralogy of the Earth - The Earth's core iron and iron alloys. Treatise on Geophysics, Vol.2: Mineralogy of the Earth, pp. 
91-120

[51] Wahr, J.M, Zuber, M.T., Smith, D.E., Lunine, J.I, 2006. Tides on Europa, and the thickness of Europa's icy shell. J. Geophys. Res. 111, E12005.

[52] Webb, E. K, Stevenson, D. J., 1987. Subsidence of topography on Io. Icarus. 70, 348-353.

[53] Xu, S., Crossley, D., Szeto, A.M.K, 2000. Variations in length of day and inner core differential rotation from gravitational coupling. Phys. Earth Planet. Int. 117, 95-110. 


\section{TABLES}

\begin{tabular}{lccccccc}
\hline & $R[\mathrm{~km}]$ & $\bar{\rho}\left[\mathrm{kg} \mathrm{m}^{-3}\right]$ & $C_{22}$ & $\frac{I}{M R^{2}}$ & $\frac{B-A}{M R^{2}}$ & e & $A_{\theta}[\mathrm{m}]$ \\
\hline Io & 1821.6 & 3527.5 & $558.8 \times 10^{-6}$ & 0.378 & $2.22 \times 10^{-3}$ & 0.0041 & $\sim 269$ \\
Europa & 1565.0 & 2989.0 & $131.5 \times 10^{-6}$ & 0.346 & $5.26 \times 10^{-4}$ & 0.0094 & $\sim 134$ \\
Ganymede & 2631.2 & 1942.0 & $38.26 \times 10^{-6}$ & 0.312 & $1.53 \times 10^{-4}$ & 0.0013 & $\sim 10$ \\
Callisto & 2410.3 & 1834.4 & $10.2 \times 10^{-6}$ & 0.354 & $4.08 \times 10^{-5}$ & 0.0074 & $\sim 12$ \\
\hline Table B.1 & & & & & & &
\end{tabular}

Mean Radius, mean density, second-degree gravity field coefficient, mean moment of inertia, equatorial flattening, eccentricity and rigid libration amplitude for the four Galilean satellites 


\begin{tabular}{|c|c|c|}
\hline layer & Thickness[km]/Radius[km] & Density $\left[\mathrm{kg} \mathrm{m}^{-3}\right]$ \\
\hline \multirow[t]{2}{*}{ ice shell } & $h_{s}=\{25,37.5, \ldots, 75\},\{100,125, \ldots ., 200\}$ & $\{800,900, \ldots, 1200\}$ \\
\hline & $\{250,300, \ldots, 400\}, 500$ & \\
\hline ocean & $h_{o}=6.2-706.2$ & $\{800,900, \ldots$, \\
\hline ice mantle & $R_{i}=\{1900,2050, \ldots, 2500\}$ & $\{1000,1100, \ldots, 1400\}$ \\
\hline rock mantle & $R_{r}=\{1500,1600, \ldots, 2000\}$ & $3000-3799$ \\
\hline fluid core & $R_{f}=\{450,700, \ldots, 1200\}$ & 996 \\
\hline solid core & $R_{c}=\{200,450, \ldots, 950\}$ & $5500,6500,8000$ \\
\hline
\end{tabular}

Table B.2

The ranges of size and of density of the six internal layers of the Ganymede models consistent with the given constraints on mass, radius and mean moment of inertia. The densities of the rock mantle and of the fluid core are calculated for the given values of the other interior parameters. Within a given set denoted by curly brackets, the values are always equally spaced. 


\begin{tabular}{lll} 
layer & Thickness $[\mathrm{km}] /$ Radius $[\mathrm{km}]$ & Density $\left[\mathrm{kg} \mathrm{m}^{-3}\right]$ \\
\hline ice shell & $h_{s}=\{5,7.5, \ldots, 15\},\{20,30, \ldots, 100\}$ & $\{800,900, \ldots, 1200\}$ \\
& $h_{o}=10-160$ & $\{800,900, \ldots, 1200\}$ \\
ocean & $R_{r}=\{1400,1425, \ldots, 1475\}$ & $3001-3785$ \\
& & \\
cock mantle & $R_{c}=\{100,200, \ldots, 800\}$ & $5151-7998$
\end{tabular}

Table B.3

Size and of density of the four internal layers of the Europa models consistent with the given constraints on mass, radius and mean moment of inertia. The ocean thickness and the densities of the rock mantle and the core are calculated for the given values of the other interior parameters. Within a given set denoted by curly brackets, the values are always equally spaced. 


\begin{tabular}{|c|c|c|}
\hline layer & Thickness $[\mathrm{km}] /$ Radius $[\mathrm{km}]$ & Density $\left[\begin{array}{ll}\mathrm{kg} & \left.\mathrm{m}^{-3}\right]\end{array}\right.$ \\
\hline \multirow[t]{2}{*}{ ice shell } & $h_{s}=\{25,37.5, \ldots, 75\},\{100,125, \ldots, 200\}$ & $\{800,900, \ldots, 1200\}$ \\
\hline & \multicolumn{2}{|l|}{$250,300, \ldots, 400$} \\
\hline ocean & \multicolumn{2}{|l|}{$h_{o}=12.7-421$} \\
\hline ice-rock interior & $R_{\text {in }}=1964-2148$ & $2299-2373$ \\
\hline \multicolumn{3}{|l|}{ Table B.4 } \\
\hline \multicolumn{3}{|c|}{$\begin{array}{l}\text { Size and of density of the three internal layers of the Callisto models consisten } \\
\text { with the given constraints on mass, radius and mean moment of inertia. The ocear }\end{array}$} \\
\hline \multicolumn{3}{|c|}{ with the given constraints on mass, radius and mean moment of inertia. The ocear } \\
\hline \multicolumn{3}{|c|}{ thickness and the density of the interior are calculated for the given values of the } \\
\hline \multicolumn{3}{|c|}{ other interior parameters. Within a given set denoted by curly brackets, the value } \\
\hline
\end{tabular}




\section{FIGURES CAPTIONS}

Fig. 1: Reference frame centered at the mass center of the satellite.

Fig. 2: Internal structure of the generic satellite consisting of six layers. The subscripts used in the text and associated with the different layers are given between brackets.

Fig. 3: Schematic representation of the different orientations of the internal regions in a model with five layers.

Fig. 4: Density profile of the interior structure of a selected model of Ganymede

Fig. 5: Amplitude of the Ganymede shell libration at the equator, $R g_{s}$, as a function of the ice shell thickness, $h_{s}$, for all our models of the internal structure of Ganymede. For each value of $h_{s}$, the values of $g_{s}$ are divided into five groups, corresponding to the five considered values of $\rho_{o}-\rho_{s}(0,100,200,300$ and $400 \mathrm{~kg} \mathrm{~m}^{-3}$ ) among our range of models. For each group, we have represented the mean value of $g_{s}$ (solid lines). The bottom and top lines correspond to $\rho_{o}-\rho_{s}=0$ and 400 , respectively.

Fig. 6: Amplitude of the Europa shell libration at the equator, $R g_{s}$, as a function of the ice shell thickness, $h_{s}$, for all our models of the internal structure of Europa. For each value of $h_{s}$, the values of $g_{s}$ are divided into five groups, corresponding to the five considered values of $\rho_{o}-\rho_{s}(0,100,200,300$ and 400 $\mathrm{kg} \mathrm{m}^{-3}$ ) among our range of models. For each group, we have represented the mean value of $g_{s}$ (solid lines). The bottom and top lines correspond to $\rho_{o}-\rho_{s}=0$ and 400 , respectively. 
Fig. 7: Amplitude of the Europa shell libration at the equator, $R g_{s}$, as a function of the ice shell thickness, $h_{s}$, away from the resonance with the free libration. For each value of $h_{s}$, the five groups corresponding to the values of $\left(\rho_{o}-\rho_{s}\right)$ can more easily be distinguished than in the previous figure.

Fig. 8: Amplitude of the Callisto shell libration at the equator, $R g_{s}$, as a function of the ice shell thickness, $h_{s}$, for all our models of the internal structure of Callisto. For each value of $h_{s}$, the values of $g_{s}$ are divided into five groups, corresponding to the five considered values of $\rho_{o}-\rho_{s}(0,100,200,300$ and 400 $\mathrm{kg} \mathrm{m}^{-3}$ ) among our range of models. For each group, we have represented the mean value of $g_{s}$ (solid lines). The bottom and top lines correspond to $\rho_{o}-\rho_{s}=0$ and 400 , respectively.

Fig. 9: Errors on the surface layer thickness determination $\left(h_{s}\right.$ if the surface layer is an icy shell, $h_{m}$ if it is a silicate mantle) for an observational error of $1 \mathrm{~m}$ on the libration amplitude. For Ganymede, Europa and Callisto, we take $\rho_{s}=\rho_{o}$. 


\section{FIGURES}

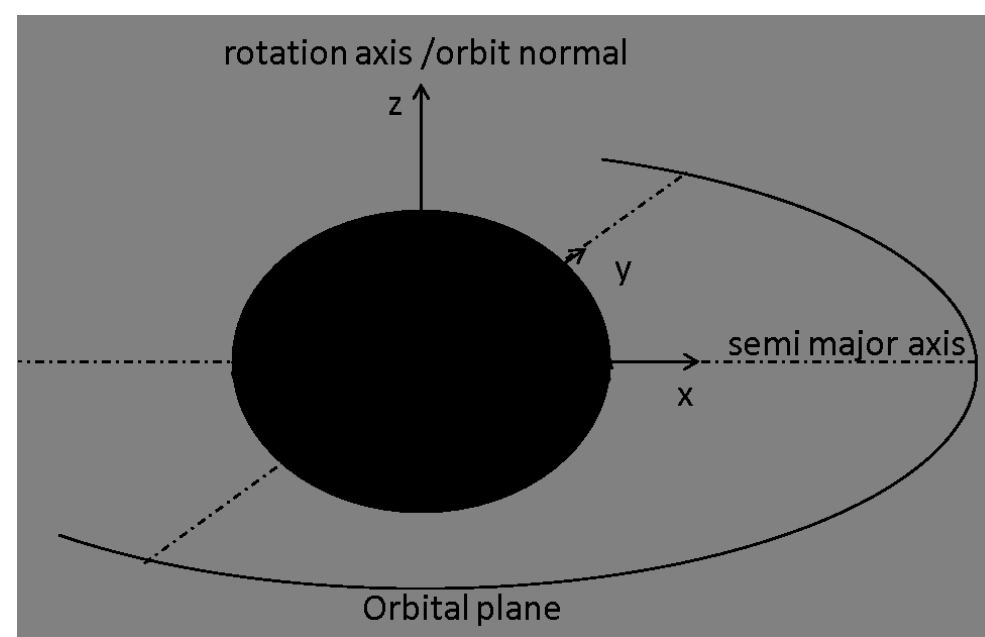

Fig. 1

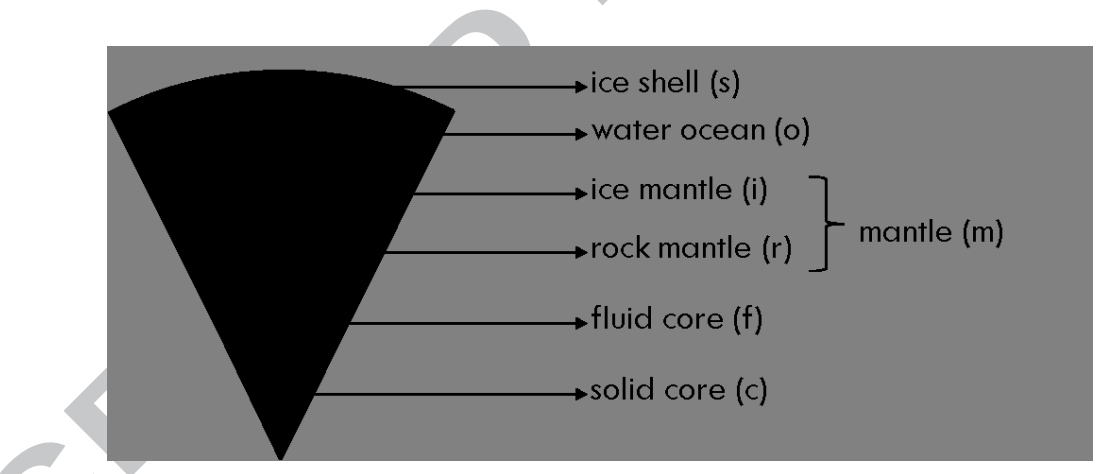

Fig. 2 


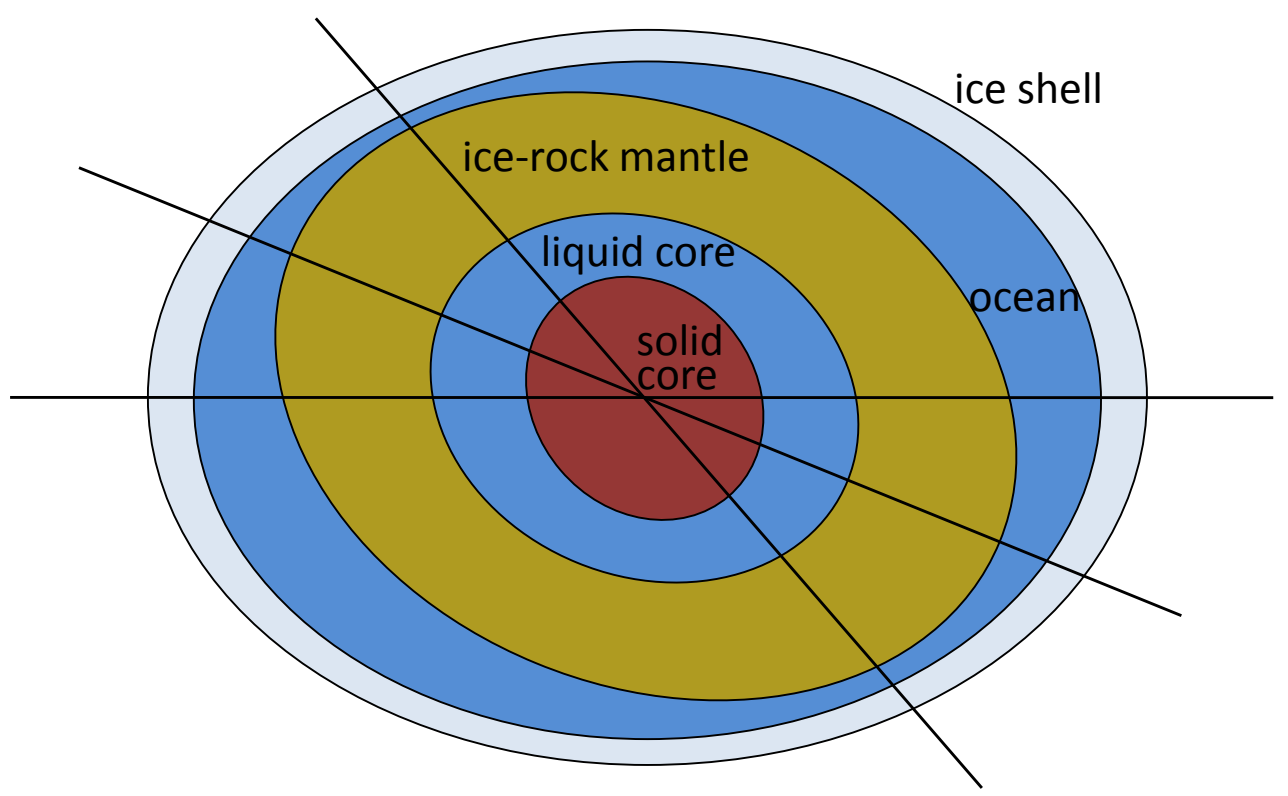

Fig. 3 


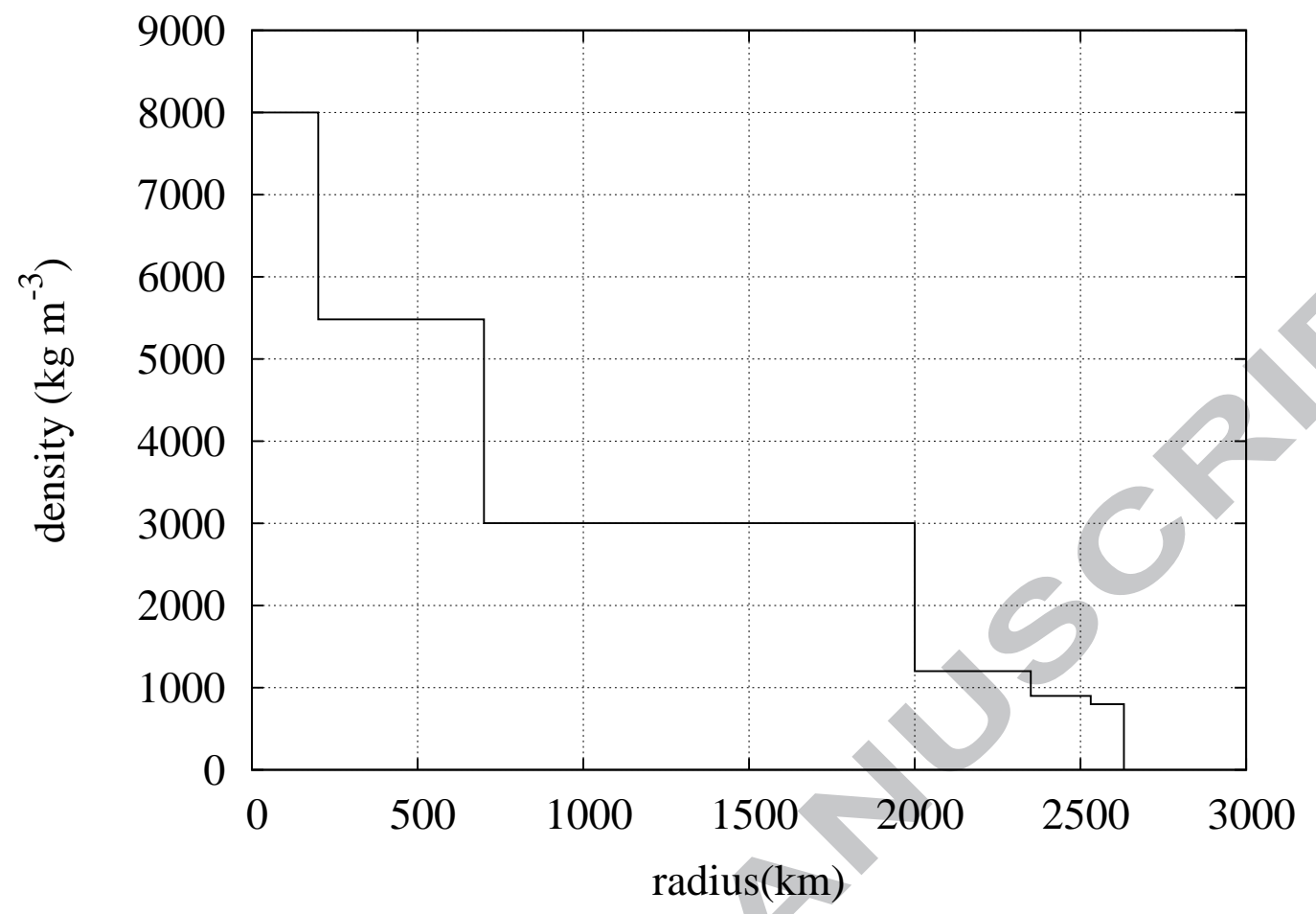

Fig. 4

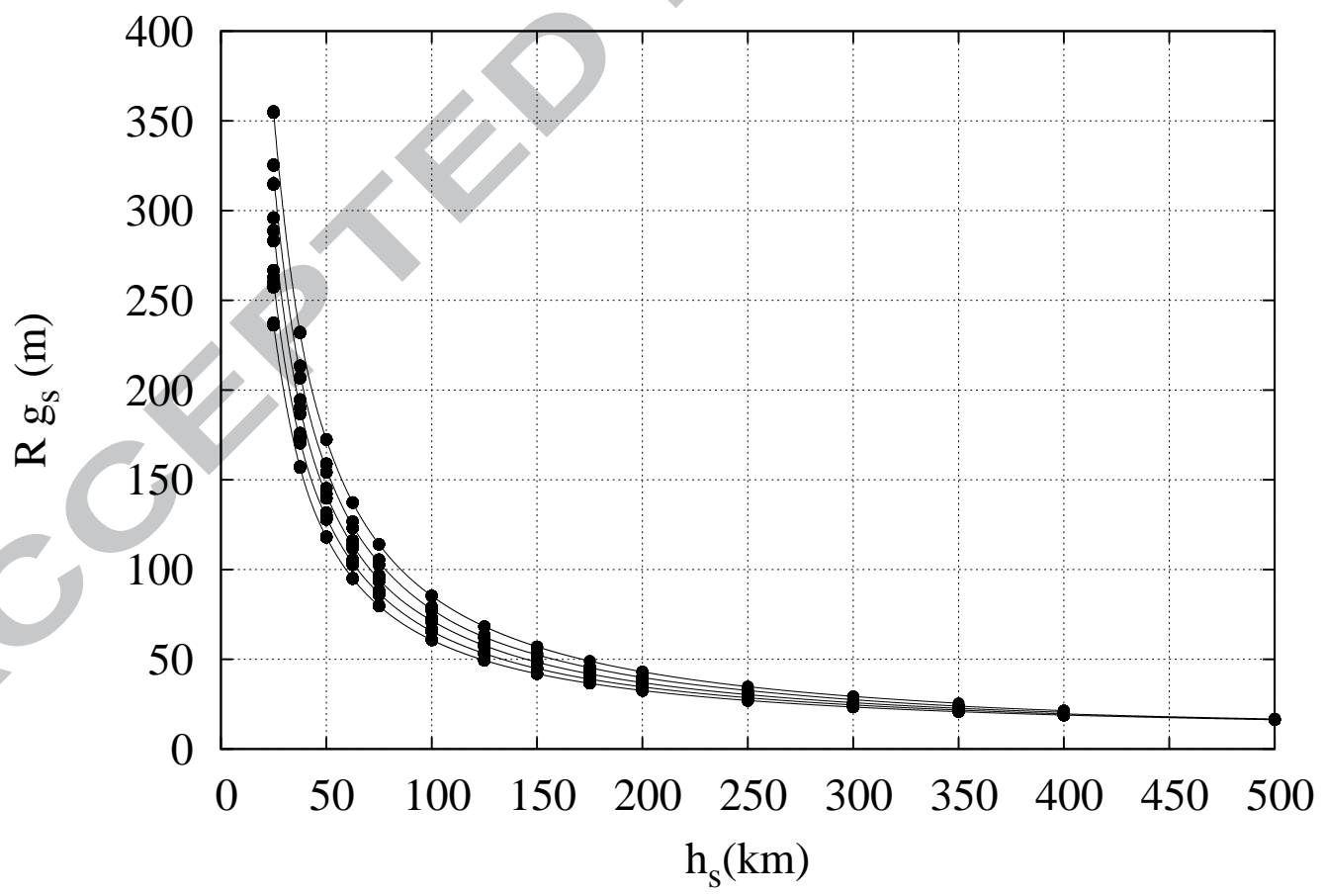

Fig. 5 


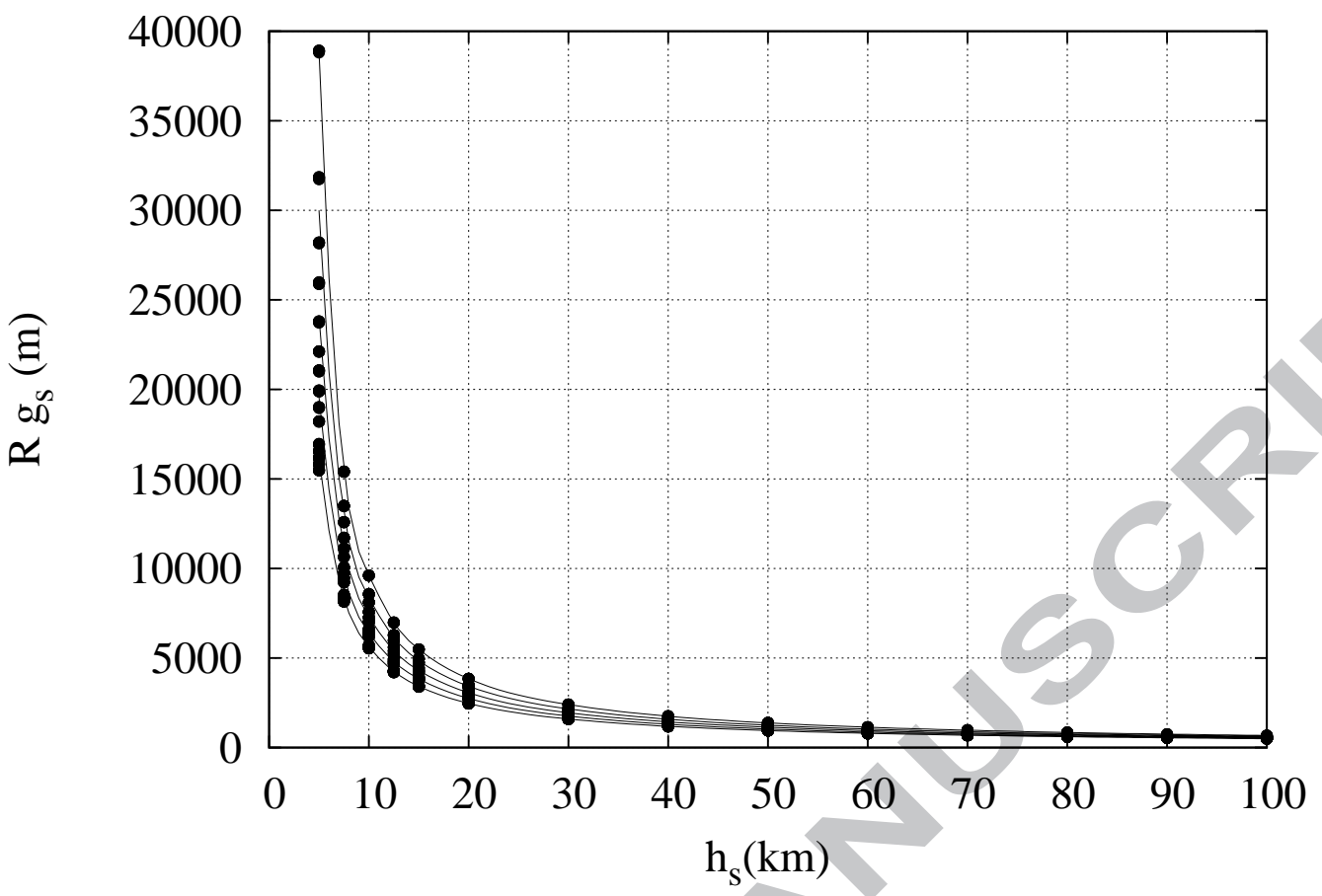

Fig. 6

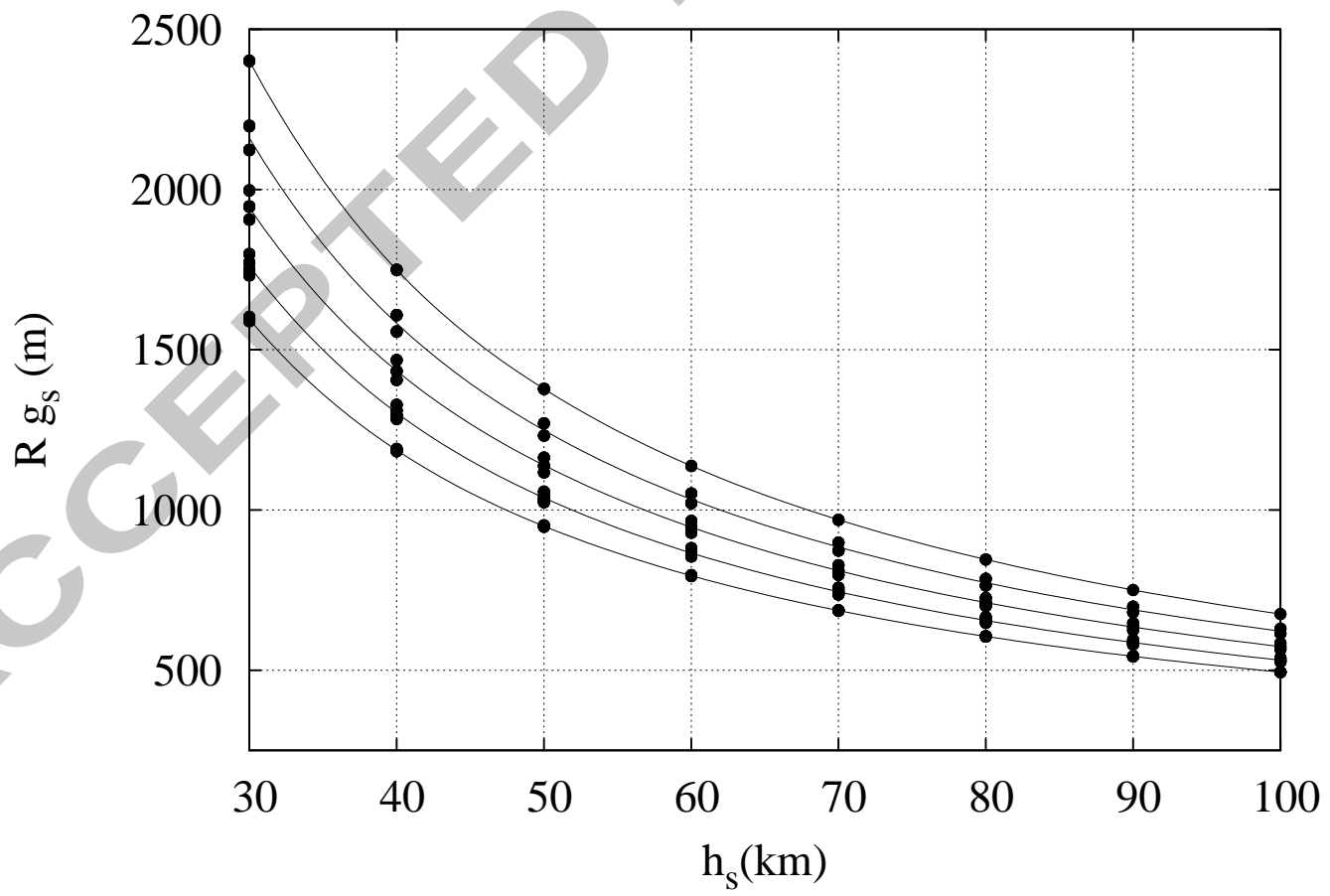

Fig. 7 


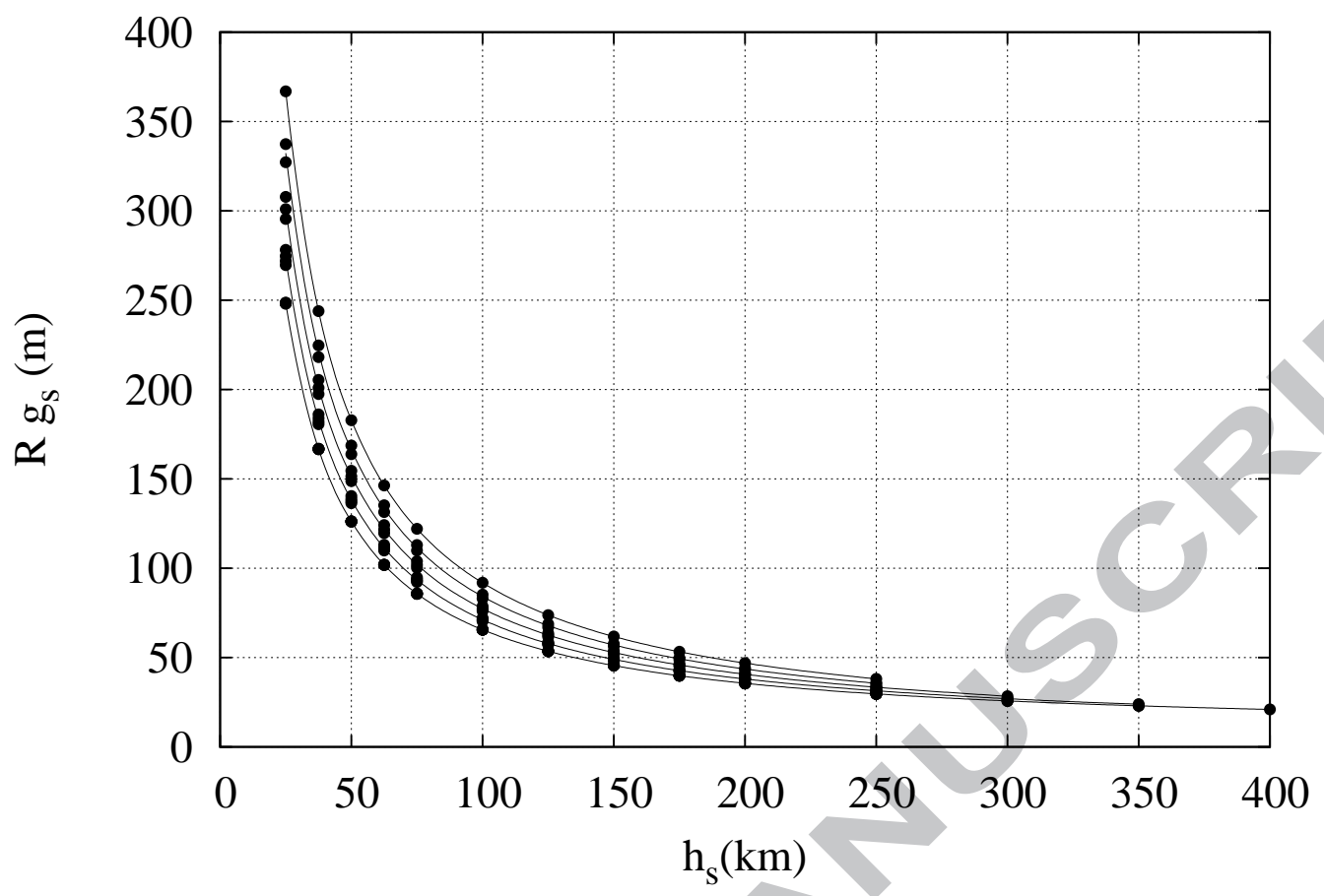

Fig. 8

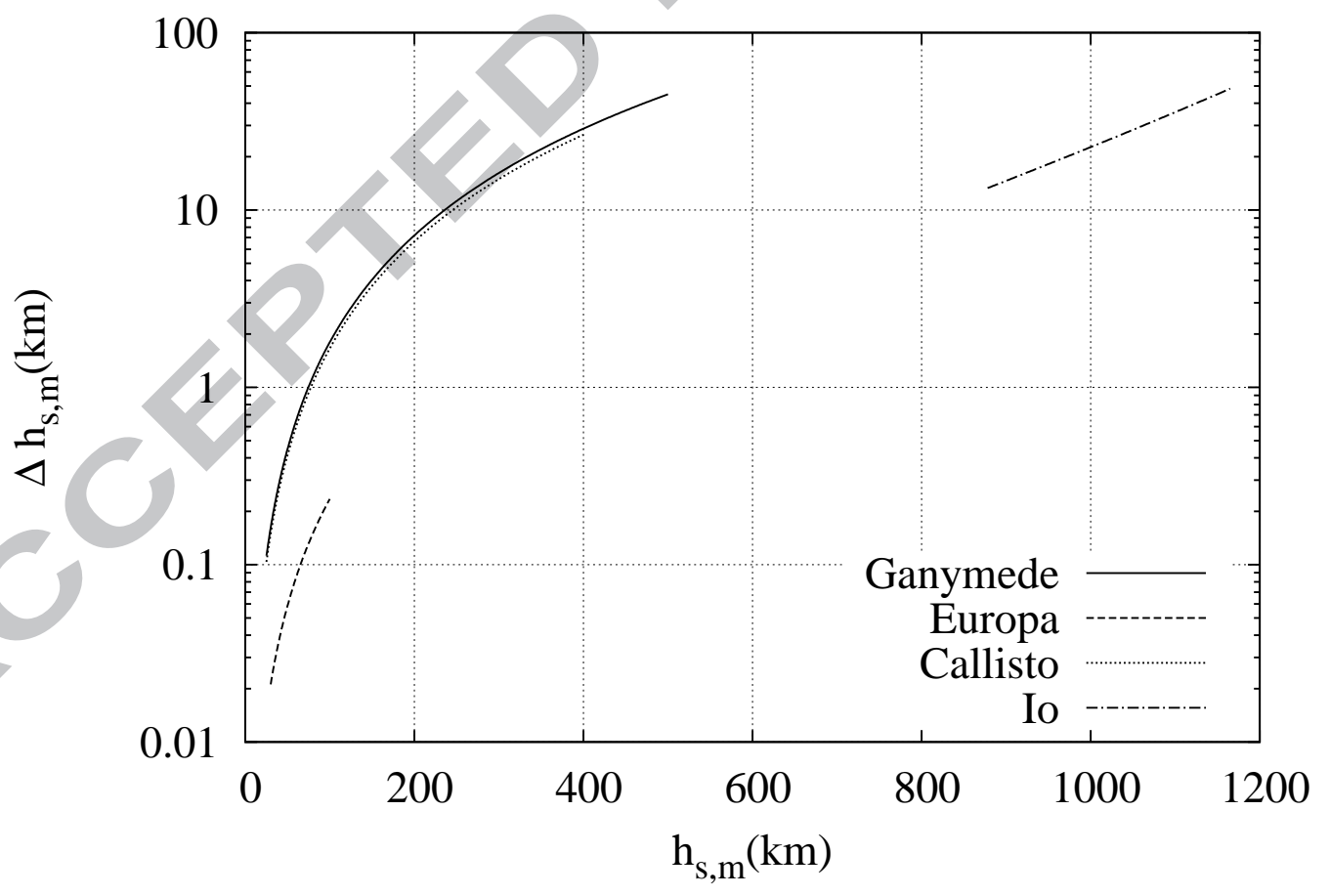

Fig. 9 